\title{
Head-to-head domain walls in magnetic nanostructures
}

\author{
M Kläui \\ Fachbereich Physik, Universităt Konstanz, 78457 Konstanz, Germany \\ and \\ Zukunftskolleg, Universität Konstanz, 78457 Konstanz, Germany \\ E-mail: Mathias.Klaebiouni-konstanz de
}

\begin{abstract}
A review of geometrically conf ned $180^{\circ}$ head-to-head domain walls is presented. The spin structures of head-to-head domain walls are systematically determined by direct imaging and magnetotransport, and quantitative domain wall type plase diagrams are obtained and compared with available theoretical predictions and micromagnetic simulations. Discrepancies to the experiment are explained by taking into account thermal excitations, and thermally-induced domain wall type transformations are observed. The coupling between domain walls via the stray f eld leads to changes in the wall spin structure and the stray feld intensity from a wall is found to decrease as $1 / r$ with distance. Using the measured stray feld values, the energy barrier height distribution for the nucleation of a vortex core is obtained. The pinning behaviour of domain walls at geometrical variations is discussed in detail and direct quantitative measurements of the width and depth of attractive potential wells responsible for the pinning are given. Dynamic measurements of resonant wall oscillations yield the exact shape of the potential well. Finally the domain wall propagation due to feld and current is brief y discussed.
\end{abstract}

\section{Contents}

1. Introduction

2. Techniques

3. Domain wall types and wall phase diagrams

3.1. Theory of head-to-head domain wall spin structures

3.2. Experimental determination of head-to-head domain wall spin structures

3.3. Thermally activated domain wall transformations

3.4. Walls in ultrathin flms

3.5. Further head-to-head domain wall lypes

3.6. Wall widths

4. Domain wall coupling energetics

4.1. Coupling between adjacent domain walls

4.2. Direct quantitative observation of the domain wall stray feld
5. Domain wall pinning

5.1. Characterization of a pinning site-potential well depth and width

5.2. Potential well curvature probed by domain wall oscillations

5.3. Furcher details of pinning behaviour

6.

6. Domain wall propagaion

6.1. Field-induced wall motion

6.2. Cument-induced wall motion

\section{Conclusions}

Acknowledgments

References

\section{Introduction}

9 Recently the plyysics of surfaces, interfaces and nanostructures has become one of the major areas of research, due to the trend 11 in science and technology towards miniaturization of physical 
systems into the nanoscale. From the scientif $\mathrm{c}$ viewpoint, such systems pose a whole new set of problems, both theoretical and experimental. Fundamentally, novel properties emerge in magnetic elements as the lateral structure dimensions become comparable to or smaller than certain characteristic length scales, such as spin diffusion length, carrier mean free path, magnetic exchange length, domain wall width, etc. The effects of the governing energy terms determine the interplay between the relevant physical length scales and the sizes of the structured materials.

When the geometry changes from the bulk to the nanoscale, the magnetic properties of ferromagnetic elements start to be governed by the element geometry and not only by the intrinsic materials properties. Such behaviour and in particular the magnetization conf gurations and reversal in small magnetic elements have been reviewed in detail for instance in [1,2]. Such a strong dependence on the geometry allows one then to tailor the magnetization conf guration and spin switching by appropriately engineering the geometry. The magnetization conf guration that constitutes the lowest energy state in a small magnetic structure can for instance be set to a multidomain state with domain walls, since the dipolar interaction (stray feld) leads to the magnetization being parallel to the element edges, which then results in a very reproducible and controllable spatially inhomogeneous magnetization distribution (domain conf guration) [1].

Domain walls, which constitute the boundary between domains, have been intensively researched in the past, though with a focus on the domain wall types that occur in the bulk or in continuous flms. The most prominent examples are the Bloch and the Néel wall types, which occur in continuous thin fi ms [3-5]. A thorough overview of such domain walls is given in [2].

In patterned magnetic structures, novel domain wall types emerge, when the wall spin structures start to be dominated by the geometry rather than by the intrinsic materials properties. This is particularly true for soft magnetic materials (and to some extent also for polycrystalline hard magnetic structures), where the effects of magnetocrystalline anisotropy and other material-dependent anisotropies are small and the element shape governs the domain wall spin structures. In soft magnetic nanostructures, the stray feld energy leads to an alignment of the magnetization with the edges of the structures, in order to minimize the stray feld. Thus, in elongated elements, such as wires, etc, the magnetization points along the long axis of the element and most often such a (quasi-)monodomain state constitutes the lowest energy magnetization conf guration [1] If such a structure is not in a monodomain state, say, for instance, two domains with opposite direction exist (see f gure $\left(\right.$ a) ), a $180^{\circ}$ head-to-head domain wall has to be present in between the domains (dotted line in f gure $1(\mathrm{a})$ ).

Theoretically, the different types of domain walls that occur in such soft magnetic wire structures have been reviewed by Thiaville in [6]. It was found that depending on the ratio between widh $W$ and thickness $t$ (see fgure I(a)), different types of domain walls occur.

For the case that the width and thickness are similar (e.g. with a circular or square cross section, $t \approx W$, which is

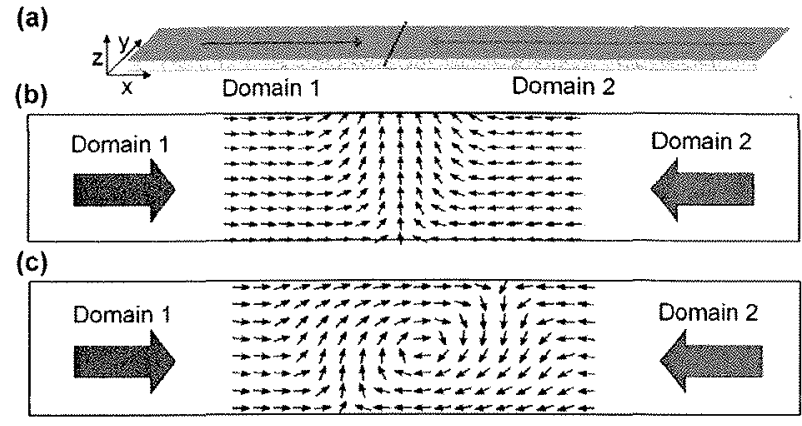

Figure 1. (a) Schematic of a magnetic wire with two domains pointing in opposite directions (red and blue arrows) and a domain wall (dotted line) separating the domains. The length of the wire is along the $x$-direction, the width $W$ along the $y$-direction and the thickness $t$ along the $z$-direction. Top view ( $x-y$ plane) of the spin structure of a transverse head-to-head domain wall (b) and a vortex head-to-head domain wall (c).

called a wire in [6]) distinct domain wall types occur as detailed in [0]. Whilst techniques exist to fabricate such wires [7], they are diff cult to make using conventional lithography (electron bean, optical, etc). Furthermore the direct imaging of domain and domain wall spin structures is diff cult due to the fact that the magnetization is often inhomogeneous across the wire.

We will concentrate therefore on 'f at' structures where the thickness is smaller than the width ( $t<W \ll$ length) and in such elements well-def ned domain wall spin structures (some of which differ from the ones occurring in square or round cross section wires) are present. In the literature, such an elongated $f$ at structure is also mostly called a "wire" and we will use this terminology since there is no danger of confusing this with 'wires' with a circular cross section (which we do not treat here). We note though, that our 'f at' geometry $(t<W)$ is called 'strip' in [6].

Domain walls in such $f$ at wires have become the focus of intense research recently for a number of reasons. Such domain walls behave as quasi-particles, which can be manipulated for instance by external magnetic fie ds, spin-polarized currents and variations in the geometry. In particular the latter, namely variations in the geometry by introducing constrictions and protrusions, yields a potential landscape, in which a quasi-particle wall can move. For elevated temperatures and large walls, the behaviour can be described within classical limits and can thus be modelled with classical micromagnetic techniques and the wall dynamics is often governed by thermal activation. At low temperatures and if a small number of spins are present, quantum behaviour such as quantum tunnelling of walls has been predicted [8] and for certain wall types, this has also been observed experimentally [9].

External magnetic felds have been used conventionally to displace walls, and wall depinning felds and velocities have been determined [10-15]. Rather than using felds, currents have been shown recently to be able to displace walls [16-18]. In general the interaction of domain walls with spin-polarized currents has become the focus of research in the past few years and from this interaction domain wall magnetoresistance results, where electrons are scattered from 
domain walls leading to a change of resistance [19-25]. Here a detailed knowledge of the domain wall spin structures is necessary, since the effects have been predicted to depend on the wall spin structure, in particular the wall width $[19,24]$. The inverse effect, namely the infl ence of spin-polarized charge carriers on the domain wall leads to current-induced domain wall motion (CIDM) induced by electrons impinging on a domain wall. The underlying spin torque effect has been the subject of rising interest, and critical current densities as well as domain wall velocities have been measured [16-18]. While there are a large number of theoretical treatments, agreement between experiment and theory is often poor and only a basic understanding exists so far [26-28]. Due to the high current densities involved in this feld of research, temperature and heating effects play a key role [29-32]; in particular, the wall spin structure was predicted to depend on temperature $[31,33,34]$.

Thus the resistivity of a domain wall, its mobility, the depinning felds and critical propagation currents due to the spin torque effect depend critically on the wall spin structure, which serves as a motivation to study these wall spin structures in detail.

In addition to fundamental physical aspects, domain walls have also been suggested for a range of applications. Sensing magnetic fie ds by domain wall displacement has been suggested [35] but domain walls have most prominently been proposed for logic and storage. Logic based on domain walls has been extensively researched and the necessary logic functions, such as NOT, AND and other gates have been developed $[36,37]$. For storage applications, domains and domain walls have been envisaged as bits, which are moved by current-induced domain wall motion for instance in a racetrack device $[38,39]$ or by feld-induced wall motion [40].

\section{Techniques}

For all these studies, high quality magnetic nanostructures have to be fabricated. The prevailing geometries to study head-to-head domain walls are straight and zig-zag wires, U-shaped, L-shaped and half-ring elements as well as full rings. Ring elements have proven to be a useful geometry for the investigation of domain walls since due to the high element symmetry, walls can be easily created and positioned by applying an external uniform magnetic feld [41, 42]. Ferromagnetic rings can be in the fux closure vortex state or in the onion state, where they exhibit $180^{\circ}$ head-to-head and tailto-tail domain walls $[41,43]$. In order to make sure that the domain walls exhibit the same behaviour in the straight parts of the structure as in the curved parts, the radius of curvature should be much larger than the width, since otherwise the wall can be pinned by the variation in the geometry (see also section 5.3 on pinning of domain walls).

In order to make these structures, a range of fabrication methods is available and details as well as reviews can be found in $[43-46]$.

To study domain walls, different methods are employed. Direct imaging of spin structures can be carried out using scanning probe techniques (magnetic force microscopy (MFM), spin-polarized scanning tunnelling microscopy, scanning Hall microscopy, etc), magneto-optical techniques (MOKE), x-ray and electron microscopy techniques. Introductory overviews can be found in [1, 2] and more detailed descriptions of the techniques can be found in various articles in [47]. The techniques used most extensively for the measurements in this review are $x$-ray magnetic circular dichroism photoemission electron microscopy (XMCD-PEEM) [48, 49]. electron holography $[50,51]$ and Fresnel imaging [52]. A very versatile, albeit more indirect method is magnetotransport, i.e. using magnetoresistive effects to determine domain wall properties [21].

In this review, we frst treat the wall types and the energetics that lead to the wall spin structure formation and the resulting wall widths. Then the interaction between domain walls is studied including direct determination of the stray feld coupling. Next the infuence of geometrical variations leading to pinning sites for domain walls is investigated and the potential landscapes are ascertained. Finally the domain wall dynamics due to felds and currents are brief y reviewed.

\section{Domain wall types and wall phase diagrams}

\subsection{Theory of head-to-head domain wall spin structures}

To understand theoretically the domain wall types and their spin structures, we need to briefl consider the energy terms that lead to the wall formation. The wall spin structure is a result of the energy minimization process (to be more precise the minimization of the appropriate thermodynamic potential, which is usually the Landau free energy (though often entropy effects are neglected and only the internal energy is considered) $[1,53]$ ). Without any externally applied felds and since we neglect anisotropies, the two important energy terms are the exchange energy, which is at the heart of ferromagnetism, and the stray feld energy due to the dipolar interaction of the spins. Qualitatively this means that if the exchange dominates, the wall should be very wide, so that there is only a small angle between adjacent spins resulting in a small exchange energy. If the stray f eld energy dominates, the spins try to stay parallel to the structure edge as much as possible, yielding a narrower wall. To go beyond such qualitative considerations, numerical calculations are necessary to ascertain the spin struciures that constitute local energy minima (stable wall structures). For the case of domain walls in wires, such micromagnetic simulations $[6,54,55]$ were carried out by McMichael and Donahue in 1997 [56]. Two wall spin structures were predicted to occur: transverse walls (TW, see figure l(b)) and vortex walls (VW, see f gure (c)). In the case of the transverse wall, the spins rotate in the plane of the structure. To reduce the energy further, the shape of the wall is asymmetric along the $y$-axis yielding a V-shaped wall (f gure $1(b)$ ) and the spins in the wall can either point in the $+y$ or $-y$ direction. The vortex wall exhibits a very different spin structure. Here the spins curl clockwise or counter-clockwise around the vortex core where the magnetization is pointing out of the plane in the $+z$ or $-z$ direction (f gure 1(c)) [57-59]. The energies of the two wall types vary with geometry and material and can be calculated 


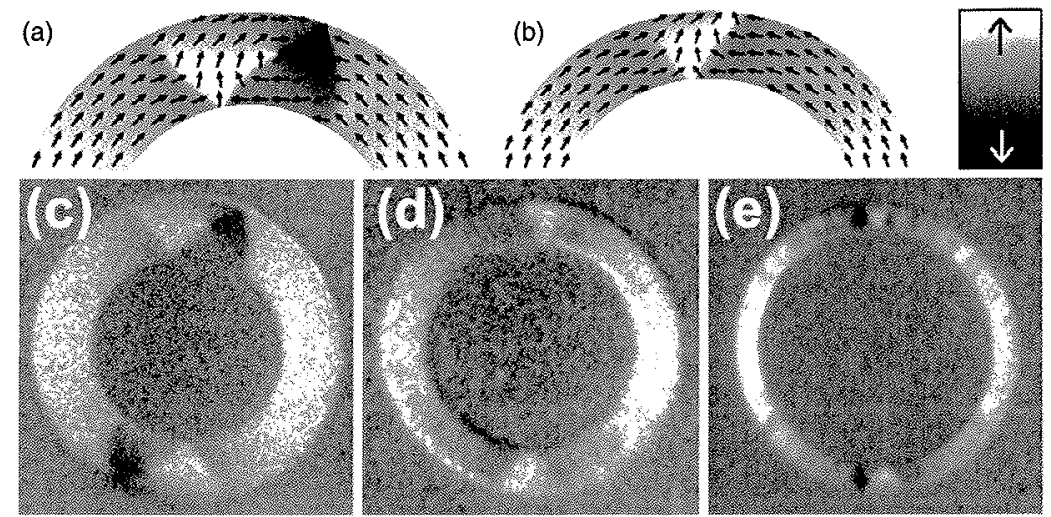

Figure 2. Spin structure of (a) a vortex and (b) a transverse wall simulated using OOMMF. PEEM images of (c) $30 \mathrm{~nm}$ thick and $530 \mathrm{~nm}$ wide (outer diameter $D=2.7 \mu \mathrm{m}$ ), (d) $10 \mathrm{~nm}$ thick and $260 \mathrm{~nm}$ wide $(D=1.64 \mu \mathrm{m})$, and (e) 3 nm thick and $730 \mathrm{~nm}$ wide $(D=10 \mu \mathrm{m})$ Permalloy rings in the onion state. The grey scale indicates the direction of magnetic contrast. Reproduced with permission from [63]. Copyright 2006, American Institute of Physics.

from the simulations. More instructive though is an analytical calculation of the energies of the two wall types as a function of geometry as carried out by McMichael and Donahue [56]. They assumed that as a frst approximation, the difference in stray feld energies between the two wall types is effectively the stray feld of the transverse component in the TW, which is less present in the VW. They calculate this stray feld energy difference to be

$$
\Delta E_{\text {stray feld }} \approx-{ }_{8}^{1} \mu_{0} M_{\mathrm{s}}^{2} t^{2} W
$$

with $M_{\mathrm{s}}$ the saturation magnetization, $t$ the thickness and $W$ the width of the structure [56]. For the difference in exchange energies they assume that it is given by the vortex in the VW, which yields

$$
\Delta E_{\mathrm{ex}}=\approx 2 \pi t A \ln \frac{r_{\max }}{r_{\min }}
$$

with $A$ the exchange constant, $t$ the thickness, $r_{\max }$ the outer radius of the vortex, which is assumed to be half the strip width and $r_{\text {min }}$ the inner radius of the vortex, which is given by the vortex core radius $\delta$. From this we can now deduce a 'phase diagram' where the energetically favourable wall type is determined as a function of the geometry (width, thickness). In particular to obtain the phase boundary. which delineates the region where one wall is favoured or the other, the sum of the energy differences is set to zero (both wall types have the same energy). Neglecting the weak logarithmic dependence this yields $W t \approx$ const, with the constant depending on the material, which means that in a width versus thickness diagram the phase boundary is a hyperbola.

These calculations were later refi ed by Nakatani and Thiaville [60] and they found, in addition to symmetric transverse walls, tilted transverse walls that constitute the energy minimum in a small range of geometries and such tilted transverse walls were actually observed experimentally [61] (for details see f gure 9 ).

These micromagnetic simulations were carried out in the $0 \mathrm{~K}$ limit but the inf uence of thermal excitations on the wall spin structure has also been investigated theoretically. Such simulations are diff cult, since a small cell size (ideally an atomistic lattice) is necessary to reproduce a realistic spin wave spectrum. So only few results have been made available and analytical calculations as well as simulations on small systems have yielded a transition to a linear wall close to the Curie temperature $[31,33]$, while more intricate behaviour is expected for the more complex head-to-head wall spin structures.

\subsection{Experimental determination of head-to-head domain wall spin structures}

To study the domain wall types experimentally $[62,63]$, arrays of $5 \times 5$ polycrystalline $\mathrm{Co}$ and Permalloy $\left(\mathrm{Ni}_{80} \mathrm{Fe}_{20}\right)$ rings with different thicknesses and widths were fabricated as described in $[46,64]$. For the investigation of the phase diagram, the edge-to-edge spacing between adjacent rings was more than twice the diameter to prevent dipolar interactions which might otherwise inf uence the domain wall type. To determine the spin structure of the domain walls as a function of the ring geometry, the samples were imaged using XMCD-PEEM [48].

In f gure 2, we present PEEM images of (c) a thick and wide Permalloy ring, (d) a thin and narrow ring, and (e) an ultrathin ring measured at room temperature. The contrast of the images is explained in (a) and (b). The domain wall type was systematically determined from PEEM images for more than 50 combinations of ring thickness and width for both Permalloy and $C o$ and the quantitative phase diagrams shown in f gures $3(\mathrm{a})$ and (c) were extracted (a similar phase diagram was also obtained in [65]). The phase diagrams exhibit two phase boundaries indicated by solid lines between vortex walls (thick and wide rings, squares), transverse walls (thin and narrow rings, discs), and again vortex walls for ultrathin rings.

We discuss $\mathrm{f}$ rst the upper boundary shown in f gures 3(a) and $(c)$. This phase boundary was investigated theoretically by McMichael and Donahue as described above [56]. The theoretical phase boundary (dotted lines) is shifted to lower thickness and smaller width compared to the experimental boundary (solid lines in f gures $3(b)$ and (d)). This discrepancy can be understood by taking into account the following: the calculations [56] compare total energies and therefore 

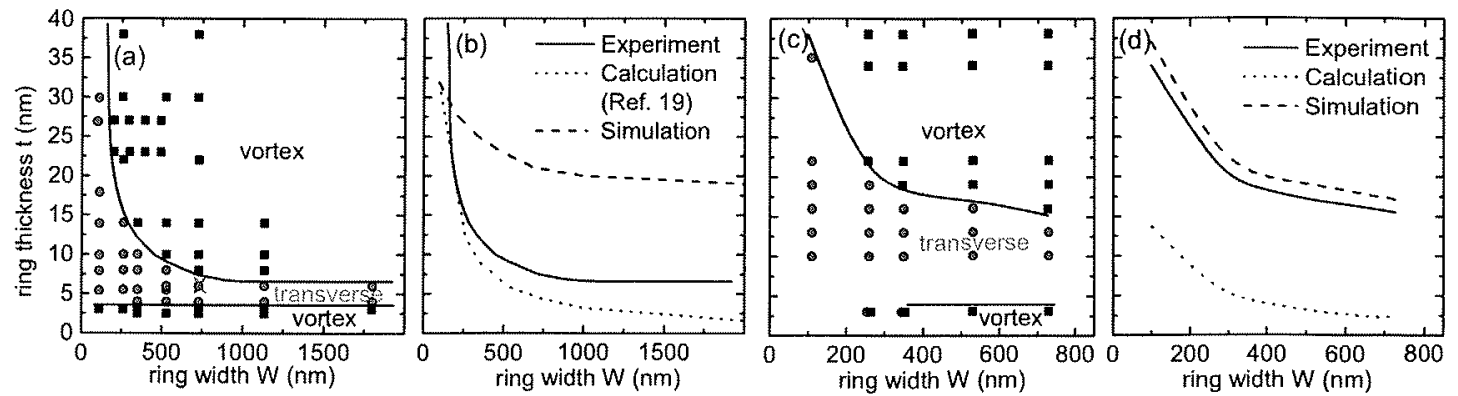

Figure 3. Experimental phase diagrams for head-to-head domain walls in (a) Permalloy and (c) Co rings at room temperature. Black squares indicate vortex walls and red discs transverse walls. The phase boundaries are shown as solid lines. ((b), (d)) Comparison of the upper experimental phase boundary (solid lines) with results from calculations according to [56] (dotted lines) and micromagnetic simulations (dashed lines). The thermally activated wall transitions shown were observed for the ring geometry marked with a red cross in (a) $(W=730 \mathrm{~nm}, t=7 \mathrm{~nm})$. Reproduced partly with permission from $[62,63]$. Copyright 2004, 2006, American Institute of Physics.
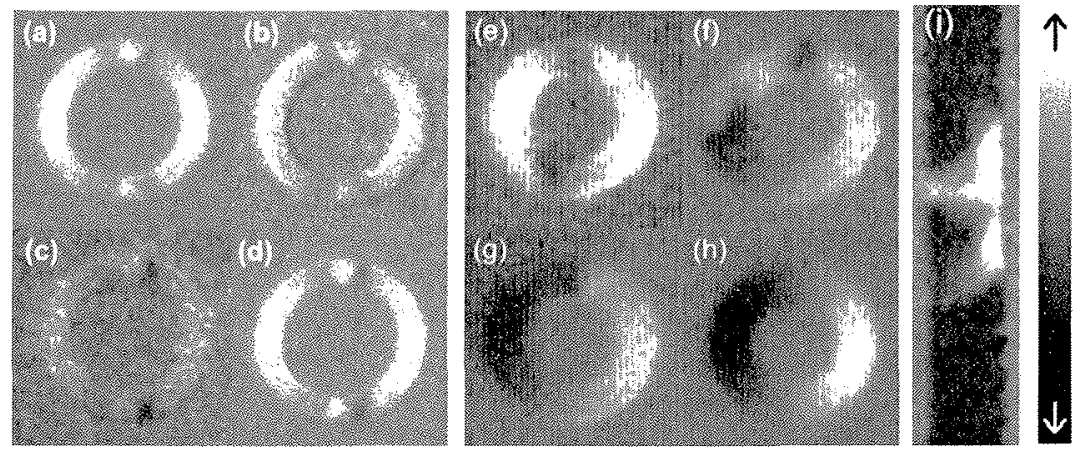

Figure 4. PEEM images of a $7 \mathrm{~nm}$ thick and $730 \mathrm{~nm}$ wide ring imaged during a heating cycle at temperatures of ((a), (d)) $T=20^{\circ} \mathrm{C}$ (be fore and after heating, respectively). (b) $T=260^{\circ} \mathrm{C}$, and (c) $T=310^{\circ} \mathrm{C}$ (estimated errors are $\pm 10 \mathrm{~K}$ ). Due to heating, rings (here (e) $7 \mathrm{~nm}$ thick, $1135 \mathrm{~nm}$ wide with two vortex walls) can attain either (g) a vortex state with a $360^{\circ}$ domain wall [69] or (h) the vortex state. The intermediate state, where one wall is displaced, is shown in (f). In (i) an effective $360^{\circ}$ wall made up of two vortex walls is shown enlarged ( $1500 \mathrm{~nm}$ wide and $20 \mathrm{~nm}$ Py wire). The grey scale indicates the direction of magnetic contrast. Reproduced partly with permission from [63]. Copyright 2006, American Institute of Physics

determine the wall type with the absolute minimum energy as being favourable. In the experiment, the wall type was investigated after saturation of the ring in a magnetic feld and relaxing the feld to zero. During relaxation, frst a transverse wall is formed reversibly [66]. For the formation of a vortex wall, an energy barrier has to be overcome to nucleate the vortex core, which leads to a hysteretic behaviour of the wall formation. So the observed spin structure does not necessarily constitute the absolute minimum energy, but transverse walls can be observed for combinations of thickness and width where they constitute local energy minima even if a vortex wall has a lower energy for this geometry. Next we have simulated the experiment by calculating the domain wall spin structure after reducing an externally applied feld stepwise using the OOMMF code [67] (for permalloy: $M_{\mathrm{s}}=$ $800 \times 10^{3} \mathrm{~A} \mathrm{~m}^{-1}, A=1.3 \times 10^{-11} \mathrm{~J} \mathrm{~m}^{-1}$; for $\mathrm{Co}: M_{\mathrm{s}}=$ $1424 \times 10^{3} \mathrm{~A} \mathrm{~m}^{-1}, A=3.3 \times 10^{-11} \mathrm{~J} \mathrm{~m}^{-1}$; for both: damping constant $\alpha=0.01$, cell size $2-5 \mathrm{~nm}$ ). The simulated boundary (dashed line) is shifted to higher thickness and larger width compared to the experiment. This can be attributed to the fact that thermal excitations help to overcome the energy barrier between transverse and vortex walls in case of the room temperature experiment, while they are not taken into account in the $0 \mathrm{~K}$ simulation. So we can expect that for temperatures above room temperature the upper experimental phase boundary is shifted to lower thickness and approaches the theoretical phase boundary. In other words, transverse walls formed at room temperature change to vortex walls with rising temperature.

In addition to studying domain wall spin structures in Permalloy and Co, we have also looked at the amorphous $\mathrm{CoFeB}[68]$. Here we fnd that due to the reduced saturation magnetization, transverse walls prevail for all the geometries studied (up to $1500 \mathrm{~nm}$ width and $20 \mathrm{~nm}$ thickness) [68].

Transverse and vortex walls have also been imaged by other groups mostly in $3 \mathrm{~d}$ metal structures with similar geometries to those we discuss here $[60-71]$.

\subsection{Thermally activated domain wall transformations}

In order to corroborate this explanation for the difference between the experiment on the one hand and calculations and simulations on the other hand, we have performed temperaturedependent XMCD-PEEM studies. Figure 4 shows an image series of a $7 \mathrm{~nm}$ thick and $730 \mathrm{~nm}$ wide Permalloy ring (geometry marked by a cross in fgure 3(a)) for different temperatures of ( $\mathrm{a}, \mathrm{d}) \mathrm{T}=20^{\circ} \mathrm{C}$ (before and after heating), (b) $T=260^{\circ} \mathrm{C}$, and (c) $T=310^{\circ} \mathrm{C}$. Transverse walls 


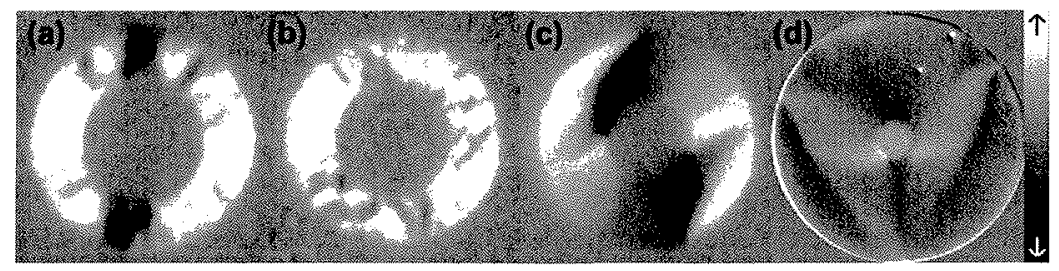

Figure 5. Limiting cases of the structures investigated: $3 \mathrm{~nm}$ thick and $1.8 \mu \mathrm{m}$ wide (a) permalloy and (b) Co rings showing ripple domain formation, (c) $6 \mathrm{~mm}$ thick and $3 \mu \mathrm{m}$ wide ring with distorted walls, and (d) $10 \mathrm{~nm}$ thick and $2.1 \mu \mathrm{m}$ wide disc-like Permalloy ring with $700 \mathrm{~mm}$ inner diameter in the triangle state (for detailed explanations of the contrast see $[73,74]$ ). The grey scale indicates the direction of magnetic contrast. Reproduced partly with permission from [97]

are formed (a) during saturation in a magnetic feld and relaxation of the feld before imaging. At $\mathrm{f} r \mathrm{st}$, heating does not infuence the spin structure of the domain walls as shown in (b), only the image contrast becomes weaker, because imaging is more diffic it at higher temperatures due to drift problems and decreasing magnetization. At a transition temperature between $T=260$ and $310^{\circ} \mathrm{C}$ corresponding to a thermal energy between $6.7 \times 10^{-21}$ and $8.0 \times 10^{-21} \mathrm{~J}$, the transverse walls change to vortex walls (c), so that a domain wall spin structure is created which is not accessible for the same ring geometry by only applying uniform magnetic fieds. Figure 4 (d) conf rms that the vortex wall is stable during cooling down. This means that both domain wall types are (meta-)stable spin conf gurations and therefore constitute local energy minima at room temperature for this geometry. These PEEM experiments directly show that the position of the upper experimental phase boundary is temperature dependent and is shifted to lower thickness and width with increasing temperature, which conf rms that both domain wall types constitute local energy minima. Experimentally, we directly observe thermally activated crossing of the energy barrier between high energy transverse and low energy vortex walls.

It should be mentioned however, that the fux closure vortex state of the ring without any domain walls and with the magnetization aligned every where along the ring perimeter is the energetically most favourable state. Many rings attain this state when the temperature is increased as shown in fgures $4(\mathrm{e})-(\mathrm{h})$ and during this process metastable $360^{\circ}$ domain walls can be formed. In order to observe the wall type transformations shown in fgures $4(a)-(d)$, it is therefore necessary that the energy barrier between transverse and vortex walls is lower than the barrier for the transition to the vortex state of the ring. This critically depends on imperfections of the ring microstructure which can serve as pinning centres and stabilize a domain wall.

It can be seen by comparing the boundaries for Permalloy and $\mathrm{Co}$ in $\mathrm{f}$ gure 3 , that for Permalloy the calculations [56] $f t$ the experiment better than the simulations while for $\mathrm{Co}$ the opposite is true. The energy barrier between a transverse and a vortex wall can be overcome more easily in the case of Permalloy rather than Co, so that transverse walls created are more likely to be retained at a certain temperature in a $\mathrm{Co}$ ring than in a Permalloy ring with analogous dimensions. This is consistent with the observation that in Permalloy there is a more abrupt change between transverse and vortex walls with varying geometry than in Co.

\subsection{Walls in ultrathin films}

We turn now to the discussion of the low thickness regime of the phase diagrams shown in f gure 3, where a second phase boundary between 3 and $4 \mathrm{~nm}$ is found both for Permalloy and for Co. In terms of energetics, this is not expected because the calculations [56] show that a transverse wall has a lower energy than a vortex wall in this thickness regime. However these calculations assume a perfect microstructure and do not take into account morphological defects such as the surface roughness. Holes, which might serve as nucleation centres for vortex wall formation, were not observed in atomic force microscopy images. However, this does not exclude a spatial modulation of magnetic properties [72] such as the exchange or the saturation magnetization, which could locally allow for a stronger twisting of adjacent spins. Thus a vortex wall would be energetically more favourable in this thickness regime only due to imperfections of the microstructure or the morphology.

In the thin samples investigated, a ripple domain formation [2] is observed as shown in fgures $5(a)$ and (b) (see also fi ure 2(e)). This can be attributed to statistical variations of the anisotropy of individual grains. Consequently, this phenomenon is more pronounced in the polycrystalline Co structures, in which individual grains exhibit a non-negligible anisotropy compared to the weak anisotropy in Permalloy.

\subsection{Further head-to-head domain wall types}

The description in the context of the phase diagrams presented here is limited to a certain geometry regime. In structures that are significa tly wider than $\approx 1 \mu \mathrm{m}$, we observe more complicated domain wall spin structures such as distorted wall spin structures (f gure $5(\mathrm{c})$ ). Wide rings with a hole in the centre exhibit a disc-like behaviour with a triangle state as shown in $f$ gure $5(d)$. This type of structure is discussed in more detail in $[73,74]$ and shows that the curvature of the element plays a crucial role if the curve radius is not much larger than the structure width.

In general, in wider structures, the inf uence of shape anisotropy is reduced and thus more complicated spin structures can constitute local energy minima and become observable. To classify these wall types, the notion explained in f gure 6 was suggested $[75,76]$ and will be used here. Apart from the simple single vortex wall (f gure 6(a)), more complicated wall spin structures including vortices with the same sense of rotation (parallel P) or opposite sense of rotation 


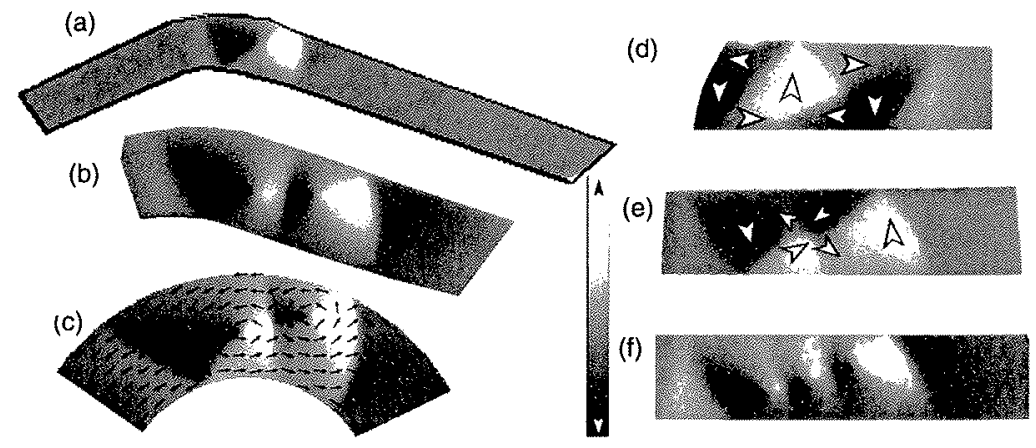

Figure 6. Further domain wall types in a $1 \mu \mathrm{m}$ wide and $28 \mathrm{~nm}$ thick Permalloy wire. The magnetization direction is given by the grey scale bar: (a) single vortex wall (VW type) located next to a kink in the wire; (b) double vortex wall with two parallel vortices and an antivortex in between (2P type); (c) micromagnetic simulation of such a $2 \mathrm{P}$ wall, visualizing the spin structure; (d) double vortex wall with two antiparallel vortices ( $2 \mathrm{AP}$ type); (e) extended vortex wall; (f) triple vortex wall with three parallel vortices and two antivortices (3P). Reproduced partly with permission from [76]. Copyright 2006, American Institute of Physics.

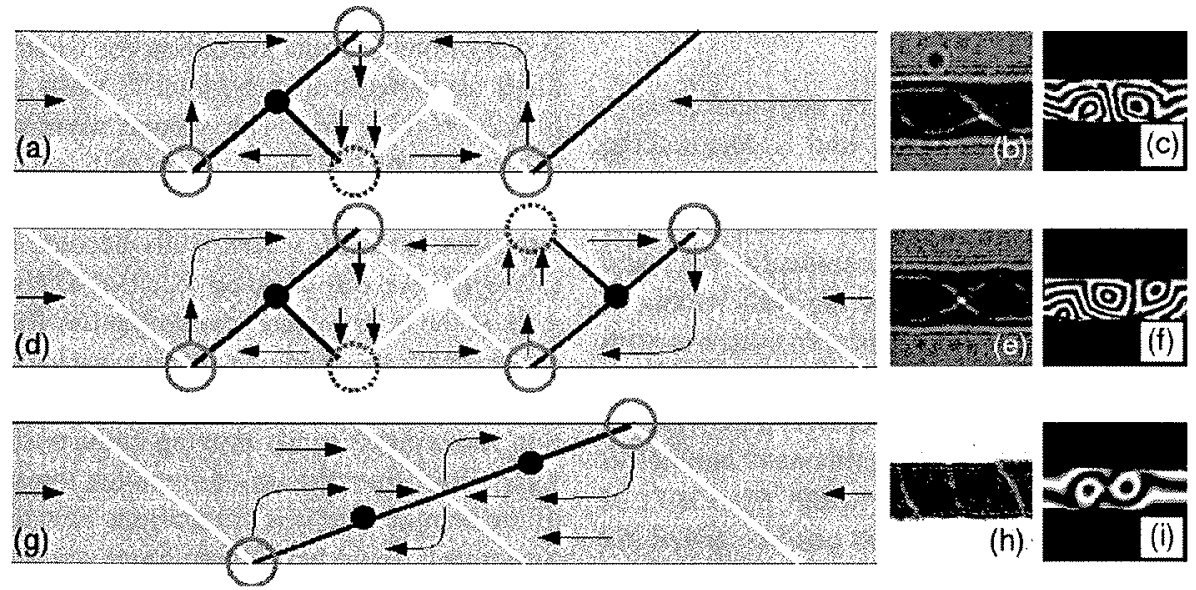

Figure 7. (a), (d), (g) Schematic spin structures, (b), (e), (h) Fresnel images and (c), (f), (i) off-axis holograms of multivortex walls taken sequentially on the same part of the sample, where (i) is a simulated image. The red (solid) and blue (dotted) circles indicate positions of opposite-sign magnetic charge accumulation. The black and white contrast in the schematic drawings corresponds to overfocus Fresnel images and for simplicity the vortex cores are drawn in the centre of the wire. The images show (a)-(c) a $2 \mathrm{AP}$ domain wall, (d)-(f) a $3 \mathrm{AP}$ domain wall and (g)-(i) a $2 \mathrm{P}$ domain wall. The contrast appearing at the sample edge is omitted in the schematic images. Reproduced with permission from [75]. Copyright 2007 Wiley-VCH Verlag GmbH \& Co.

(antiparallel AP) are found (f gures $6(\mathrm{~b})-(f)$ ). One of the reasons that such spin structures are stable in very thick structures is the magnetic stray feld that is present for single transverse or vortex walls due to magnetic (pseudo-)charges at the edge of the structure. In the case of a $2 \mathrm{AP}$ wall, some $\mathrm{fux}$ closure is achieved as shown in $f$ gure 7 . There are areas with opposite magnetic charge (denoted with blue dotted circles and red continuous circles) and if they are close, some fux closure can be achieved between them, thereby reducing the stray f eld and the associated energy. This is particularly obvious for the 2AP and the 3AP wall structures in f gures $7(\mathrm{a})$ and (d). For the $2 \mathrm{P}$ wall (f gure $7(\mathrm{~g})$ ), which is reminiscent of a cross-tie wall [2], no such fux closure is possible since the stray feld is similar to that of a single vortex wall. Observation of the double vortex wall with antiparallel vortices (2AP) was also reported using magnetic force microscopy [77], transmission electron microscopy techniques [78] and transmission $x$-ray microscopy [79]. More complicated domain wall types were also observed in [80].
In general it was found that more complicated wall spin structures ( $2 \mathrm{P}, 2 \mathrm{AP}, 3 \mathrm{P}, 3 \mathrm{AP}, \mathrm{etc})$ tend to be more susceptible to defects and irregularities compared to the simple transverse and vortex walls. One of the reasons is the fact that pinning at defects occurs preferably near the vortex core or the areas where magnetic charges are present. This is due to the fact that the energy can be lowered if vortex cores are placed at positions with reduced exchange energy (e.g. non-magnetic defects) and magnetic charges arrange themselves around edge irregularities to lower the stray feld energy. Thus to obtain reliable behaviour we will concentrate in the following on the properties of simple single transverse and vortex walls.

\subsection{Wall widths}

One of the key issues for the interaction between domain walls and spin-polarized currents is the domain wall width, since it governs whether the interaction occurs adiabatically (meaning that the conduction electron spins follow the magnetization 


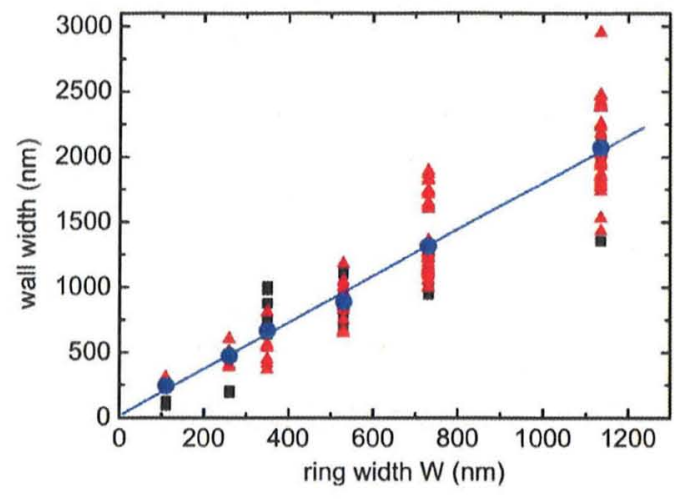

Figure 8. Domain wall width as a function of the ring width in Permalloy obtained from magnetization profi es along the ring. Red triangles represent vortex walls, black squares transverse walls, and blue discs the average values. The blue line is a linear $f t$ to the average values. Reproduced partly with permission from [97].

direction as they pass across the wall) or non-adiabatically (leading to scattering of the electrons and a mistracking of the spin compared to the wall magnetization direction). Wall widths have been previously studied for domain wall types other than head-to-head walls theoretically [81] and experimentally [82]. We here determine the wall widths of head-to-head domain walls by imaging the spin structure with high resolution using electron holography and XMCD-PEEM. The simplest method to determine an average wall width is to take the trace of the magnetization component parallel to the wire axis and then deduce the wall width from a $\mathrm{ft}$ of the profle averaged across the wire. This can be carried out for transverse and vortex walls but only gives a very rough measure of the wall width as it does not take into account the details of the actual two-dimensional spin structure. Results of this rough measure for the wall width for a large number of walls are shown in f gure 8 . Each measurement is indicated by a red triangle (vortex wall) or a black square (transverse wall). We do not observe any clear systematic difference between the widths for the different wall types for the geometries considered. The average wall width is shown by the blue discs, and scales approximately linearly with the structure width.

To investigate the wall widths in more detail, we concentrate on one wall type, namely transverse walls. We chose transverse walls since large effects due to the interaction with spin-polarized currents are expected in particular for small wall widths, and the narrow walls that occur in narrow structures are of the transverse type. To stabilize transverse walls at certain positions, constrictions down to $30 \mathrm{~nm}$ were introduced into wires as seen in $\mathrm{f}$ gure 9 .

A schematic of such magnetic elements with element width $w_{\mathrm{e}}$ and constriction width $w_{\mathrm{c}}$ is shown in fgure 9(a). The elements were fabricated using electron beam lithography on the silicon nitride $\left(\mathrm{Si}_{3} \mathrm{~N}_{4}\right)$ membrane substrates required for off-axis electron holography observations as described in [45]. In agreement with micromagnetic predictions of symmetrical and asymmetrical walls [60], we experimentally f nd symmetrical transverse walls (f gure 9(b)), asymmetrical tilted (f'gure 9(c)) and asymmetrical buckled transverse DWs (f gure 9(d)). Figure 9(e) shows the percentage of each wall type in different constriction ranges. In line with the results in [60] we fnd symmetric walls only in narrow constrictions and asymmetric walls prevailing for wider constrictions. Both tilted asymmetric and symmetric walls appear in the range of $w_{\mathrm{c}}<175 \mathrm{~mm}$. The second asymmetric wall type has a buckled shape (f gure $9(\mathrm{~d})$ ) and can be found for large constrictions, $w_{\mathrm{c}}=75-367 \mathrm{~nm}$. Since in thick samples with large constrictions, vortex walls become the most favourable DW type, we can consider buckled DWs as an intermediate state at the onset of the transformation from a transverse wall to a vortex wall where the vortex core has not yet nucleated.

As seen in fi ures 9(b)-(d), the magnetization conf guration varies signif cantly moving in the positive $y$-direction (see f gure 9(a)) from the tip of the notch towards the outside edge of the element and it is not obvious how the DW width should be def ned. To go beyond the rough approach used in f gure 8 , we make use of the fact that all the transverse walls exhibit a triangular spin structure. From the analysis of the TW spin structures we $f$ nd that the opening angle $\alpha_{\text {DW }}$ of the triangular transverse walls as shown in $f$ gure 9 (b) proves to be a suitable quantity to characterize most DWs. After determining $\alpha_{\mathrm{DW}}$, an average DW width $w_{\text {DW }}$ could be calculated according to

$$
w_{\mathrm{DW}}=w_{\mathrm{c}} \tan \left(\alpha_{\mathrm{DW}} / 2\right) \text {. }
$$

Experimentally we now observe an increase of $\alpha_{\mathrm{DW}}$ from $85^{\circ}$ for $w_{\mathrm{c}, \text { avg }}=50 \mathrm{~nm}$ to $100^{\circ}$ for $w_{\mathrm{c}, \text { avg }}=300 \mathrm{~nm}$ (f gure $10(\mathrm{a})$ ). By calculating $w_{\text {DW }}$, we fnd an increase from 50 to $380 \mathrm{~nm}$

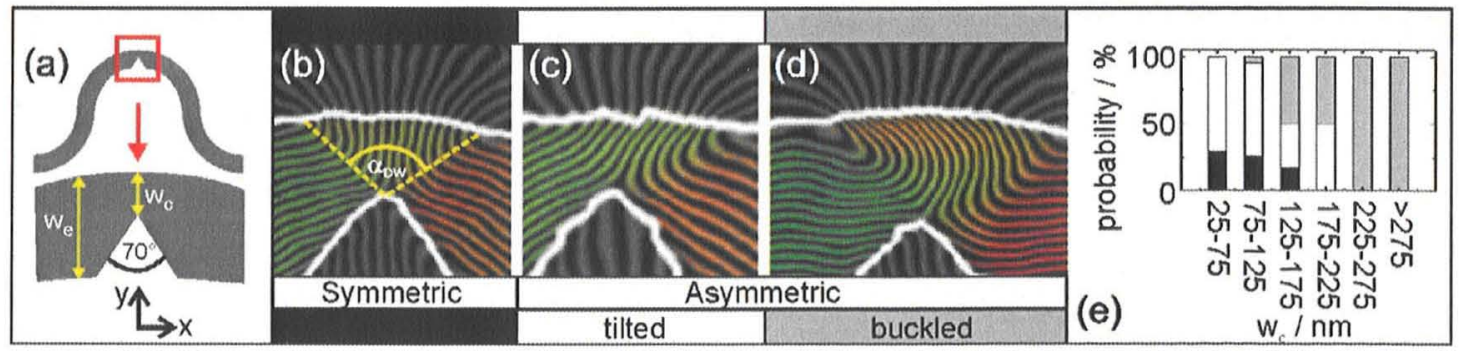

Figure 9. (a) Schematic of the Permalloy element geometry, with element width $w_{\mathrm{e}}$, constriction width $w_{\mathrm{c}}$, and notch angle of $70^{\circ}$. The DW opening angle $\alpha_{\mathrm{Dw}}$ is shown in (b). (b-d) Off-axis electron holography images of the observed transverse DW types with 11 nm thickness; $w_{\mathrm{c}} / w_{\mathrm{e}}: 138 \mathrm{~nm} / 400 \mathrm{~nm}$ (b), $103 \mathrm{~nm} / 300 \mathrm{~nm}$ (c), $191 \mathrm{~nm} / 300 \mathrm{~nm}$ (d). (e) Distribution in given ranges of $w_{\mathrm{c}}$ of transverse DW types; symmetric (black), asymmetrical tilted (white), asymmetrical buckled (grey). Reproduced with permission from [61]. Copyright 2007, American Institute of Physics. 

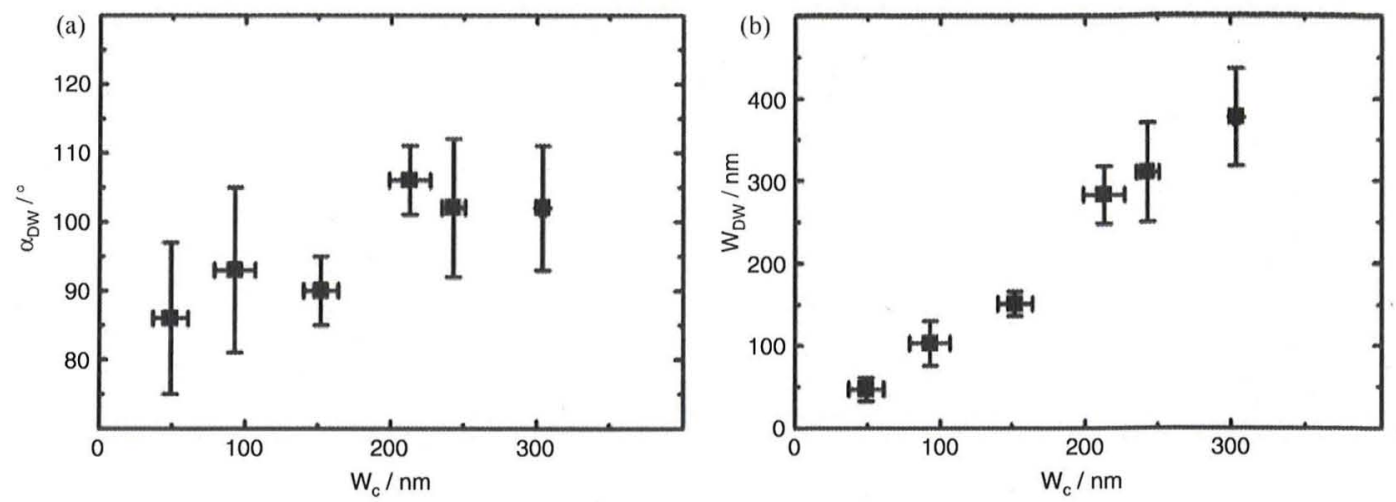

Figure 10. (a) Dependence of DW angle $\alpha_{\text {DW }}$ on an averaged constriction width $w_{c, \text { arg }}$ obtained from experiments. (b) DW width $w_{\text {DW }}$ calculated using the DW angles $\alpha_{\text {DW }}$ from (a). Reproduced partly with permission from [61]. Copyright 2007, American Institute of Physics

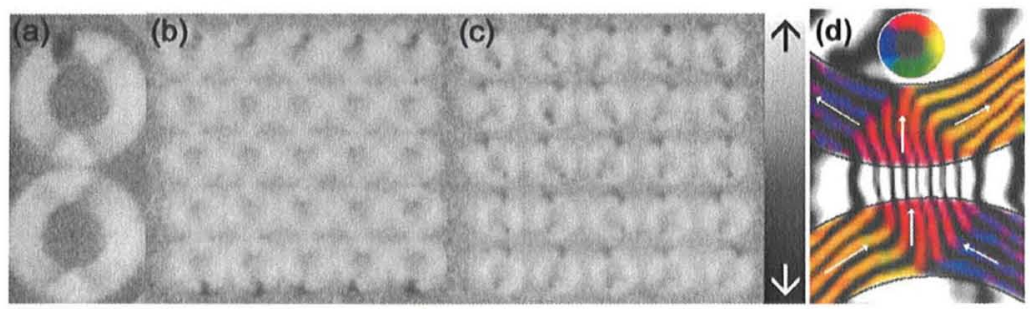

Figure 11. (a) A high-resolution XMCD-PEEM image of two rings in the onion state, after saturation with an external f eld in the vertical direction and subsequent relaxation. White and black contrasts correspond to the magnetization pointing up and down, respectively. A non-interacting vortex wall (top) and three interacting transverse walls are visible. Overview images of an array of $27 \mathrm{~nm}$ thick and $350 \mathrm{~nm}$ wide Permalloy rings with an edge-to-edge spacing of (b) $40 \mathrm{~nm}$ and (c) $500 \mathrm{~nm}$, respectively. The transition from $100 \%$ transverse walls inside the array for narrow spacings (b) to close to $0 \%$ for large spacings (c) can be clearly seen. Since domain walls at the top and bottom edges of the array do not interact with adjacent walls, they are vortex walls for all spacings investigated. (d) Electron holography inage of two interacting transverse walls. The strong stray f eld coupling is visible from the high density stray f eld fux lines between the two walls Reproduced partly with permission from [84]. Copyright 2006, American Institute of Pliysics.

(f gure 10(b)) averaged for all the walls in a certain range of $w_{\mathrm{c}}$. No signif cant inf uence of the element width $w_{\mathrm{e}}$ and the thickness of the material on $\alpha_{\mathrm{DW}}$, and therefore $w_{\mathrm{DW}}$, could be found for the range of thicknesses $(5-20 \mathrm{~nm})$ and $w_{\mathrm{e}}(100-$ $400 \mathrm{~nm}$ ) considered. Similarly, in f gure 8 no signif cant inf uence of the thickness on the wall widths was observed for the thickness range where well-def ned TWs and VWs are present.

The key energy contributions to the DWs are the exchange energy, which favours large wall widths, and the stray feld energy (shape anisotropy), which favours alignment of the spins parallel to the element edges. The increasing inf uence of the stray feld energy results in smaller $w_{\text {DW }}$ for smaller constrictions. The increase in the opening angle means that $w_{\text {DW }}$ according to equation (3) increases more than linearly with increasing $w_{\mathrm{c}}$ and this bodes well for obtaining very narrow domain walls in very narrow constrictions.

\section{Domain wall coupling energetics}

Arrays of $350 \mathrm{~nm}$ wide rings with edge-to-edge spacings down to $10 \mathrm{~nm}$ were fabricated to investigate the different dipolar coupling strengths between domain walls in adjacent rings and the resulting domain wall types. For the transmission offaxis electron holography experiments, 3/4-rings were patterned from $27 \mathrm{~nm}$ thick Co flms on $50 \mathrm{~nm}$ thick SiN membranes as detailed in [83]. Open rings rather than full rings were grown on the fragile membranes in order to facilitate the liftoff process which cannot be assisted by ultrasound. In order to obtain quantitative information about stray felds, Co samples were investigated by off-axis electron holography. Co was chosen in addition to Permalloy for this investigation due to its higher saturation magnetization and the resultant higher stray feld.

\subsection{Coupling between adjacent domain walls}

After saturating with a magnetic feld and then relaxing the fie d, rings attain the onion state characterized by two head-tohead domain walls as shown before (see also f gure 11(a)) [84]. An array of 25 rings in the onion state exhibits 50 walls in total. The domain walls inside the array interact with adjacent walls via their stray felds. Only 10 walls. which are located at the two opposite edges (top and bottom edges in f gures 11 (b) and (c)) of the array and therefore have no neighbouring rings, are not infl enced by stray felds of an adjacent wall. For all experiments, ring thickness and width were chosen such that isolated rings of this geometry exhibit vortex walls according to the phase diagram presented above.

Figure 11 shows XMCD-PEEM images of arrays of $27 \mathrm{~nm}$ thick Permalloy rings with (b) $40 \mathrm{~nm}$ and (c) $500 \mathrm{~nm}$ edge- 

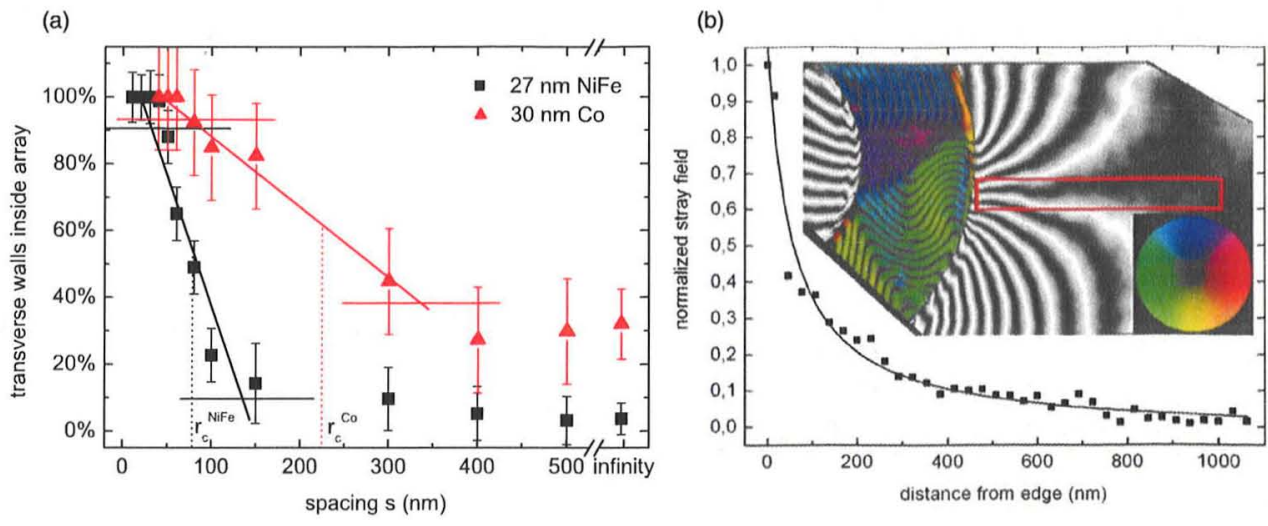

Figure 12. (a) Percentage of transverse walls inside a ring array as a function of edge-to-edge spacing. Black squares are for 27 nm Permalloy, and red triangles for $30 \mathrm{~nm}$ Co, respectively. The error bars represent the absolute statistical error $1 / \sqrt{ } n$ due to the f nite number $n$ of domain walls investigated. The horizontal lines show the 10-90\%-levels of the transition from a transverse to a vortex domain wall. (b) The inset shows an off-axis electron holography image of a transverse wall in a $27 \mathrm{~nm}$ thick Co $3 / 4$-ring. The colour code indicates the direction of the in-plane magnetization and the black lines directly represent the stray feld. The stray fie $\mathrm{d}$ strength was measured at several distances inside the marked area. The data points show the stray feld normalized to the saturation magnetization as a function of the distance $r$ from the ring edge for the wall shown in the inset. The line is a $1 / r$-fi. Reproduced with permission from [84]. Copyright 2006, American Institute of Physics.

to-edge spacing, respectively, as well as a high-resolution image (a) presenting both wall types. Vortex walls can be easily identif ed by black and white contrast which occurs because all magnetization directions corresponding to the full grey scale are present in a vortex (fi ure II(a), top). In contrast, transverse walls exhibit the characteristic grey-whitegrey contrast with the triangular spin structure in their centre (f gure 11(a), bottom). In (d) the stray feld coupling is directly visualized by the fux lines between the two domain walls.

In $f$ gure 12(a), we show the percentage of transverse walls inside the array as a function of the edge-to-edge spacing for $27 \mathrm{~nm}$ thick Permalloy rings (black squares) extracted from images of the type shown in f gure 11. A decreasing number of transverse walls is found with increasing spacing. Domain walls at the edges of the arrays are vortex walls, irrespective of the spacing, due to the absence of dipolar coupling with adjacent walls. The data points for infi ite spacings in $\mathrm{f}$ gure 12 (a) result from these domain walls. The transverse to vortex transition is characterized by a (10-90\%)width of the switching distribution of $w=(65 \pm 9) \mathrm{nm}$ and a centre at $r_{\mathrm{c}}=(77 \pm 5) \mathrm{nm}$. In f gure 12(a), red triangles show a similar transition for $30 \mathrm{~nm}$ thick Co rings with $w=(328 \pm 130) \mathrm{nm}$ and $r_{\mathrm{c}}=(224 \pm 65) \mathrm{nm}$.

In order to explain these results, we frst consider the process of domain wall formation in an isolated ring. When relaxing the applied external feld from saturation, transverse walls are initially formed. In order to create a vortex wall, a vortex core has to be nucleated. This hysteretic transition from one wall type to the other involves overcoming a local energy barrier [66], since the nucleation of the vortex core is associated with a strong twisting of the spins in the core region [85]. In arrays of interacting rings, the edge-to-edge spacing-dependent stray feld stabilizes transverse walls so that for small spacings (corresponding to a strong stray feld from the adjacent domain wall) transverse walls are favoured (f gure $11(b)$ ). For increasing spacing, the inf uence of the stray fie d from an adjacent wall is reduced, until vortex walls are formed in the rings with the lowest energy barrier for the vortex core nucleation. The more the spacing increases the more rings nucleate vortex walls (f gure 11(c)). Thus the spacing at which a wall switches from transverse to vortex is related to the nucleation barrier, which depends on local imperfections such as the edge roughness. So the number of domain walls that have switched from transverse to vortex as a function of the edge-to-edge spacing is a measure of the distribution of energy barriers for the vortex core nucleation.

For Permalloy, a relatively sharp transition occurs from all walls being transverse to all walls being vortex walls. This corresponds to a narrow energy barrier distribution, while the domain walls in Co rings exhibit a much wider transition. This difference is thought to result from the different polycrystalline microstructures of the Permalloy (magnetically soft fec crystallites with negligible anisotropy) and the Co (hep crystallites with strong uniaxial anisotropy leading to a larger number of pinning sites). Furthermore, this results in the presence of transverse walls in our Co sample even at infi ite spacings. Thus, we chose Co for the electron holography measurements rather than Permalloy in order to be able to observe a transverse wall and its stray feld in an isolated structure (see next section 4.2).

The interaction between rings can change not only the wall type, but it can also lead to changes in the switching properties if the switching process involves domain walls [1]. In particular the switching f elds of rings have been determined as a function of the spacing and, depending on the switching mechanism, an increase or a decrease of the switching felds was found with increasing spacing [86]. Furthermore the interaction leads to a narrowing of the switching feld distribution, which points to collective switching [86]. This means that due to the coupling the reversal of a single ring will lead to a reversal of the neighbouring ring so that the switching propagates along a row of rings. Such collective switching was directly observed by MFM imaging [71]. 

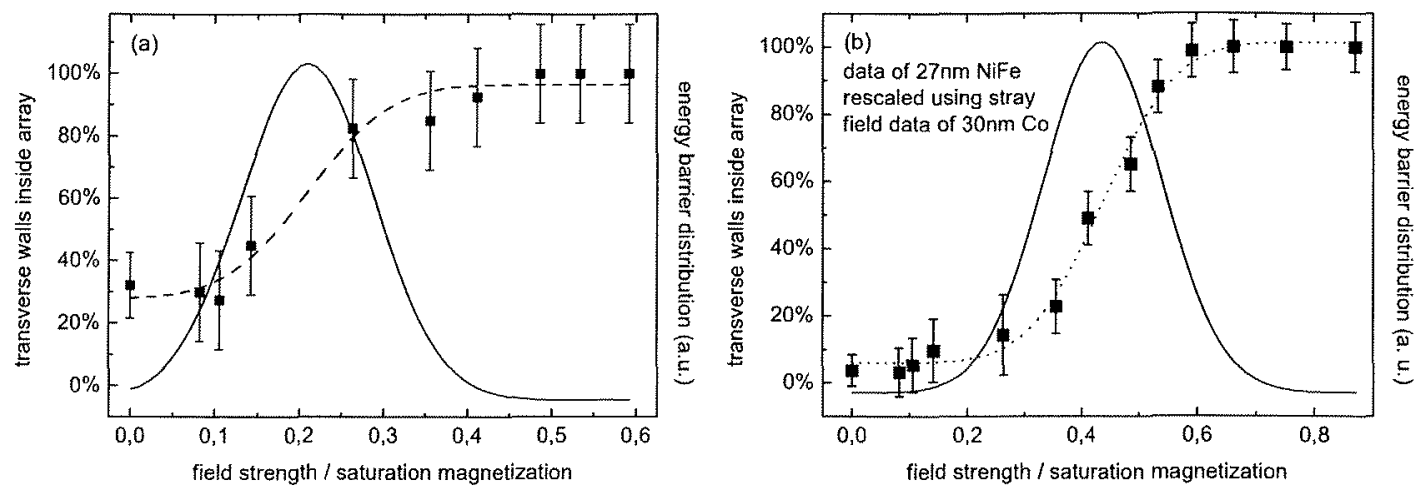

Figure 13. (a) Black squares represent the same data points as shown in figur 12 (a) for the $30 \mathrm{~nm}$ thick Co sample, but as a function of the normalized fie $\mathrm{d}$ strength. The black dashed line shows a $\mathrm{f}$ with the error function. The corresponding Gaussian distribution of the energy barriers is shown as a full red line. (b) Corresponding data for the $27 \mathrm{~nm}$ thick Permalloy sample rescaled using the stray feld measurement of a domain wall in $30 \mathrm{~nm}$ thick Co. Reproduced partly with permission from [84]. Copyright 2006, American Institute of Physics.

\subsection{Direct quantitative observation of the domain wall stray field}

The spacing-dependent distribution for the vortex core nucleation needs to be transformed to a distribution as a function of the stray feld strength, which is to a frst approximation proportional to the energy. To do this, the stray feld as well as the magnetization of the domain wall was imaged using off-axis electron holography. The inset of $f$ gure $12(\mathrm{~b})$ shows an image of the in-plane magnetic induction integrated in the electron beam direction, obtained from a transverse wall in a $27 \mathrm{~nm}$ thick isolated Co 3/4ring designed with the same width as that of the rings imaged by XMCD-PEEM. No significa $t$ difference between the functional dependence of the stray feld on the spacing is expected for a $27 \mathrm{~nm}$ and a $30 \mathrm{~nm}$ thick sample. To extract the absolute feld strength in the sample plane that acts on a neighbouring domain wall, the signal has to be rescaled to take into account the in-plane felds above and below the sample (the out-of-plane components do not contribute anyway). This can be done by calibrating the measured signal in the structure and renormalizing it to $M_{\mathrm{s}}$ (the sample thickness and saturation magnetization $M_{\mathrm{s}}$ of Co is known) [84]. The measured signal along the length of the region indicated in the image, is then equally renormalized and shown as a function of the distance $r$ from the ring edge in $f$ gure $12(b)$. The line is a $1 / r$-fi which can be expected for the distance dependence of the stray f eld created by an area of magnetic poles for small $r$ [87]. This dependence also conf rms earlier results from indirect Kerr effect measurements [86]. In order to obtain the stray feld of one single domain wall acting on an adjacent wall, the stray feld of an isolated wall was imaged.

The spacing-dependent energy barrier distribution is now rescaled to a feld-dependent distribution using the measured stray feld decay of fi ure 12(b) and presented in f gure $13(\mathrm{a})$. The rescaled data can be $f$ tted with the error function $\operatorname{erf}(x)$, which is the integral of a Gaussian distribution. Assuming a similar dependence of the stray feld for Permalloy to that measured for $\mathrm{Co}$, a Gaussian distribution is also obtained for the energy barrier height distribution for Permalloy as presented in f gure 13(b). Thus a Gaussian distribution for the energy barriers is found, which is in agreement with the presence of independent local pinning centres at the particular wall position that determine the nucleation barrier. The position of the maximum is $H_{\mathrm{max}} / M_{\mathrm{s}}=0.21 \pm 0.10$ and the full width at half maximum $w / M_{\mathrm{s}}=0.16 \pm 0.05$, where $M_{\mathrm{s}}$ is the saturation magnetization. Using $E_{\max }={ }_{2} \mu_{0} M_{\mathrm{s}} H_{\mathrm{max}}$, an energy density of $E_{\max }=(8.4 \pm 4.0) \times 10^{4} \mathrm{~J} \mathrm{~m}^{-3}$ equivalent to the f eld $H_{\max }$ can be obtained for the $30 \mathrm{~nm}$ thick Co sample.

Figure 12(a) shows that the transition for the Co sample saturates at a fnite value for large spacings. In terms of the model described above, which explains how vortex walls are formed during relaxation from saturation, this means that an additional effective feld would be needed to overcome the pinning of the remaining transverse walls at structural imperfections and to allow the vortex core nucleation and formation of an energetically favourable vortex wall. Since the pinning is much stronger in our $\mathrm{CO}$ sample than in the Permalloy sample, this occurs here only for Co.

\section{Domain wall pinning}

This section introduces the important concept of domain wall quasi-particle behaviour. Domain walls exhibit certain quasiparticle properties when for instance subjected to geometrical variations in a structure. As discussed in section 3 the detailed wall spin structure is the result of an energy minimization process and the energy terms depend not only on the material but also on the local geometry and externally applied felds. This means that geometrical variations generate a potential landscape that a domain wall feels, and by varying the geometry we can engineer this landscape. Additionally, the potential landscape can be modifed by applying external magnetic felds, so that the equilibrium position and spin structure for a wall depend on the geometry as well as the external f eld.

Intuitively, one can predict that domain walls should attain an energy minimum at a position where the wall is particularly short, since the wall energy scales as a frst approximation with 

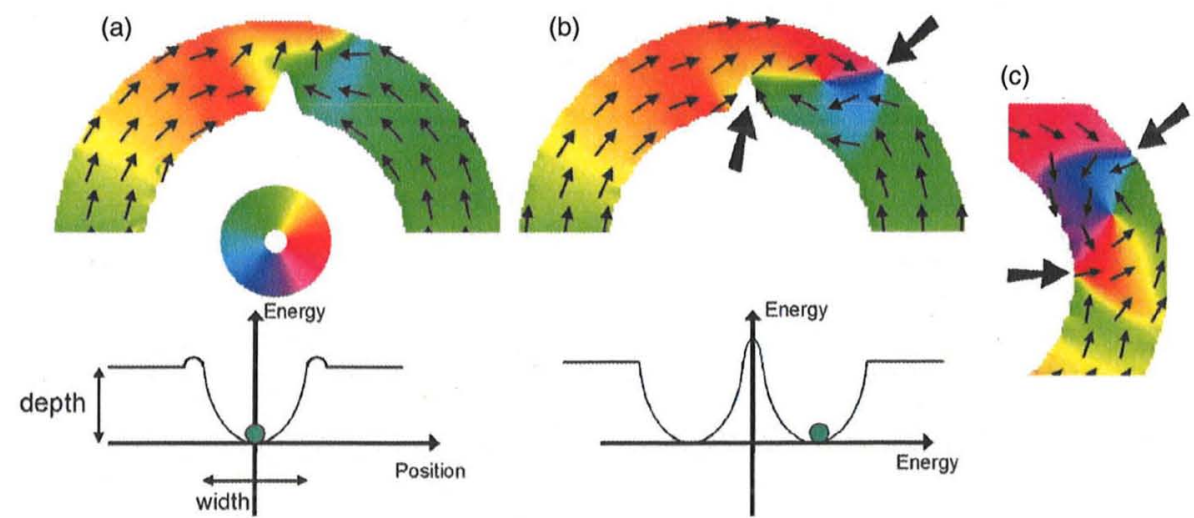

Figure 14. Simulated behaviour of a transverse and a vortex wall at a constriction. (a) shows a transverse domain wall inside a constriction and below the corresponding potential landscape schematically (single potential well at the notch position). In (b) this is shown for a vortex wall, which is pinned adjacent to the constriction. The potential landscape exhibits a double well. The black arrows in (b), (c) point to the positions where stray feld enters or leaves a vortex wall.

the size (in particular the length) of the wall so that smaller domain walls in narrower constrictions are more energetically favourable. From this we expect that a domain wall is pinned at a constriction, which thus generates an attractive potential well.

But this simple picture does not do justice to the more sophisticated aspects of domain wall behaviour, since it neglects the actual spin structure of the two domain wall types (transverse and vortex). Detailed micromagnetic simulations predict for the two wall types very different behaviour at constrictions: transverse walls are predicted to be attracted into constrictions whereas vortex walls should be repelled at the precise location of the constriction but pinned close by with a def nite sense of rotation (clockwise or counterclockwise depending on which side of the constriction the wall is located) [10.66].

In fgure 14 the potential landscape that a constriction generates is shown schematically for both transverse (a) and vortex (b) walls. We can observe that constrictions indeed create an attractive potential well for transverse walls, but vortex walls are repelled from a constriction $[10,21]$. Furthermore, the simulations predict that in general the depth of the potential well increases with decreasing constriction width, indicating that walls in narrower constrictions are more strongly pinned.

We now employ direct imaging of domain walls to reveal the details of their interaction with constrictions. In f gure 15 we present high spatial-resolution magnetic induction maps of domain valls at various constrictions, which were prepared by saturating the sample with a feld along the notch position and then relaxing the feld to zero. For narrow constrictions (f gures 15(a) and (b)) transverse walls can be discerned inside the constrictions, while for larger constrictions (f gure 15(c)(g)) vortex walls are located at positions adjacent to the constrictions (on the left or right hand side depending on slight geometrical asymmetries in the notch with respect to the applied feld direction) and this behaviour was also observed by McGrouther et al [78]. We can now directly correlate the sense of rotation with the position with respect to the

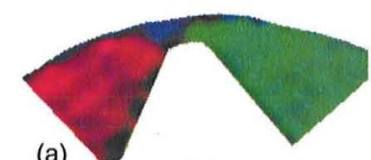

(a)
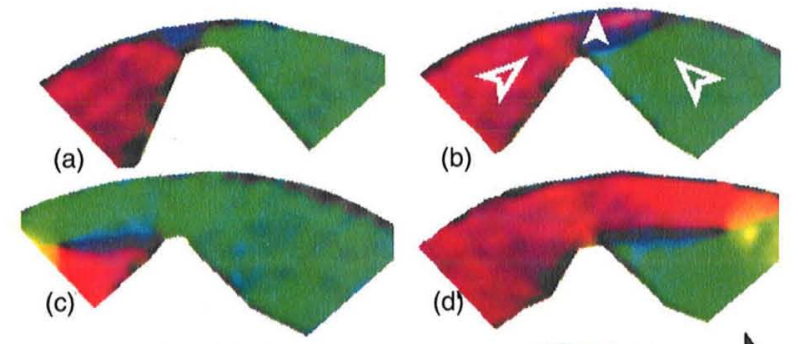

(b)
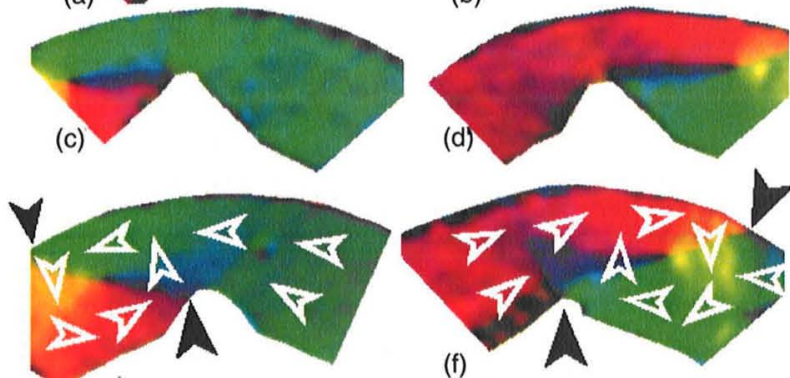

(e)
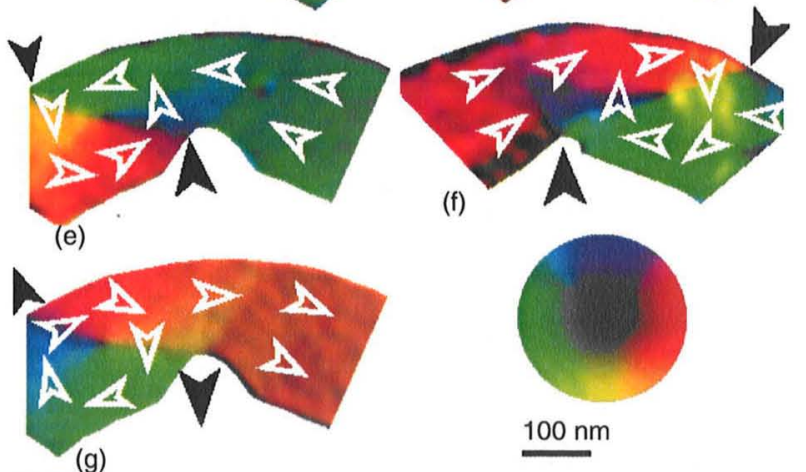

$100 \mathrm{~nm}$

Figure 15. High spatial-resolution electron holography images of sections of $27 \mathrm{~nm}$ thick, $200 \mathrm{~nm}$ wire structures with different constriction widths: (a) $35 \mathrm{~nm}$, (b) $60 \mathrm{~nm}$, (c) $100 \mathrm{~nm}$, (d) $110 \mathrm{~nm}$, (e) $140 \mathrm{~nm}$, (f) $160 \mathrm{~nm}$, and (g) $140 \mathrm{~nm}$. The walls in constrictions (a) and (b) are transverse walls, (c) and (e) exhibit vortex head-to-head walls to the left and (d) and ( $f$ ) vortex head-to-head walls to the right of the constriction; (e) and $(\mathrm{g})$ show the same constriction with (e) a head-to-head and (g) a tail-to-tail vortex wall. The magnetization directions are indicated in (b), (e), (f), and (g) by white arrows. Areas where stray fie d occurs are denoted with black arrows in (e), (f) and (g). The colour code indicating the magnetization direction is also shown. Reproduced with permission from [10]. Copyright 2005, American Institute of Physics.

notch. We see that the vortex head-to-head walls to the left of the constriction have a counter-clockwise sense of rotation (f gures $15(\mathrm{c})$ and (e)), while the walls to the right of the notch have a clockwise sense of rotation (f gures $15(\mathrm{~d})$ 


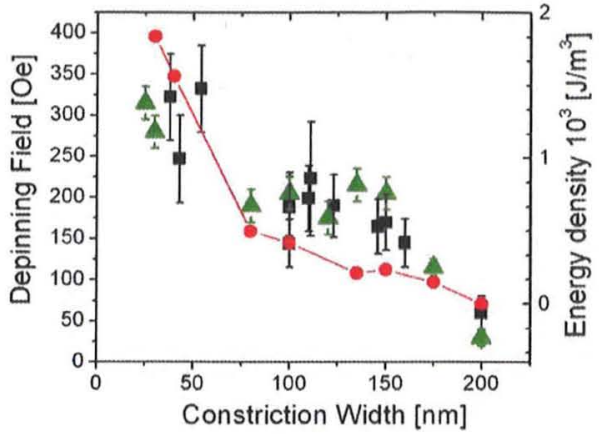

Figure 16. Experimental depinning fie d (left ordinate) versus constriction width in $200 \mathrm{~nm}$ wide Permalloy ring structures with triangular constrictions and a fi m thickness of $34 \mathrm{~nm}$ (black squares); numerical simulations (green up triangles). The calculated energy density differences show the same trend (red disks, right ordinate). Reproduced with permission from [10]. Copyright 2005. American Institute of Physics.

and ( $f)$ ). When the feld is reversed to create tail-to-tail walls, the vortex wall sense of rotation is reversed. This can be seen by comparing the vortex walls to the left of the notch in fgures $15(\mathrm{e})$ and $(\mathrm{g})$, which show the same notch after applying opposite felds. The head-to-head domain wall in (e) has a counter-clockwise sense of rotation, while the tailto-tail wall in $(\mathrm{g})$ has a clockwise sense of rotation. This is a universal behaviour, which we observed for all vortex walls at constrictions for all $\mathrm{fl} \mathrm{m}$ thicknesses. To understand this behaviour, we have to take into account the fact that a vortex wall has two areas where a stray feld occurs. In f gure 15 , the positions at which the stray feld enters (f gures 15(e) and (f)) or leaves (figur $15(\mathrm{~g})$ ) the vortex wall are marked by two black arrows. In order to minimize the stray feld associated with the vortex wall, the lower of the two stray fie $\mathrm{d}$ areas is always located inside the notch, which determines the sense of rotation of the vortex wall. As a result the wall is actually pinned in the location directly adjacent to the notch (even though it is repelled from the constriction). This behaviour explains the fact that a signif cant depinning f eld is necessary to displace the vortex walls as well as the transverse walls $[10,66]$.

\subsection{Characterization of a pinning site-potential well depth and width}

To characterize a potential well, the width, the depth and the well curvature (see f gure 14) have to be ascertained.

The depth corresponds to the pinning strength and in order to probe this, we have measured the external magnetic felds necessary to depin a domain wall from the constriction as a function of the constriction width (f gure 16) [10]. The depinning f eld of the narrowest constrictions (335 Oe) is about six times the feld needed to move a wall in a ring without a constriction $(60 \mathrm{Oe})$, where the depinning feld is governed by material imperfections and the edge roughness. Therefore the geometrically-induced pinning is by far stronger than the pinning due to natural imperfections, which means that the pinning strength can be tailored over a wide range of values by adjusting the notch geometry. Comparison to micromagnetic simulations and quantitative calculations of the depth of the potential well shows good agreement (f gure 16). Similar results for the depinning f elds have also been obtained by other groups [88].

The width (the spatial extent) of a potential well was measured in [21]. and was found to extend far beyond the physical size of the notch due to the f nite size of the domain wall. To vary the width of the potential well, the shape of the constriction can be varied, i.e. the notch can be made narrower or wider:

\subsection{Potential well curvature probed by domain wall oscillations}

Finally to fully characterize the pinning potential, the curvature has to be determined in addition to the width and the depth. For static measurements, the curvature does not play a crucial role, but if the quasi-particle domain wall is excited, one could expect that it carries out an oscillatory motion in the potential well analogous to a (harmonic) oscillator. To study these dynamics, a structure with constrictions is fabricated. An SEM image of a $25 \mathrm{~nm}$ thick and $200 \mathrm{~nm}$ wide Permalloy ring structure with $2 \mu \mathrm{m}$ diameter and 10-30 $\mathrm{nm}$ wide notches with electrical contacts, which was fabricated as described in [89], is shown in f gure 17(a).

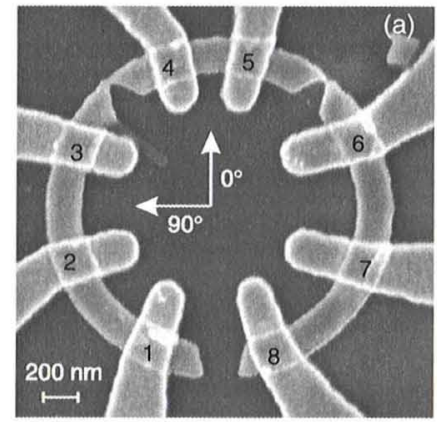

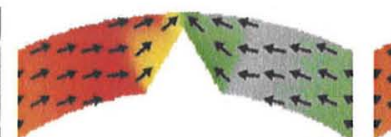

(b)

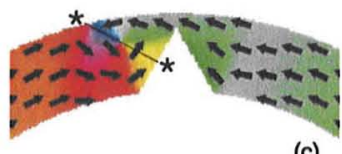

(c)
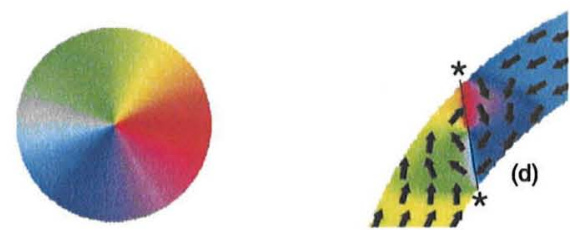

Figure 17. (a) SEM image of the device with numbered contacts. Micromagnetic simulation of a transverse wall inside the constriction (b) and of a vortex wall adjacent to the constriction (c). (d) Micromagnetic simulation of the free vortex wall. The two stars indicate the topological edge defects and the resonant mode corresponds to the vortex core moving along the line connecting the two stars. The colour circle indicates the direction of the magnetization. Reproduced with permission from [94]. Copyright 2007 by the American Physical Society 
(a)

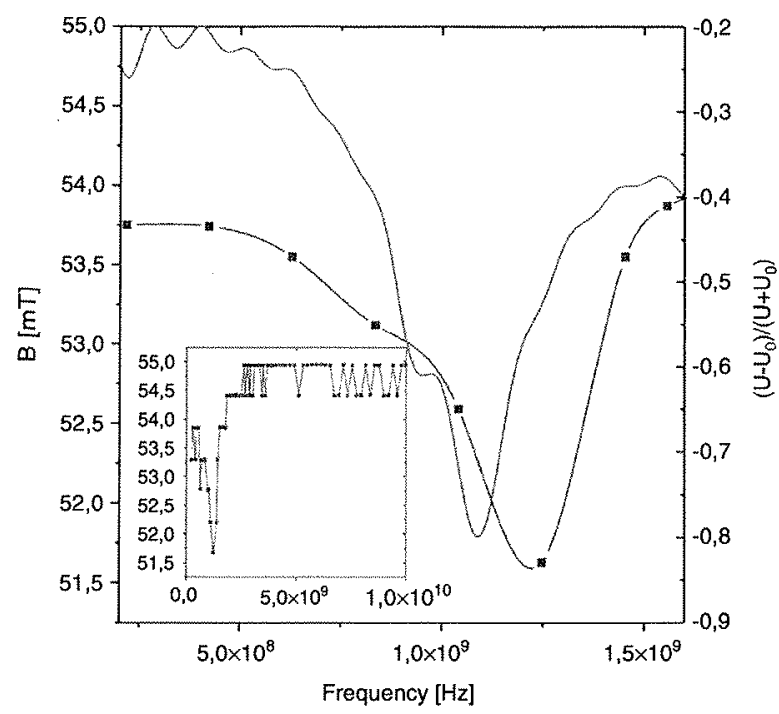

(b)

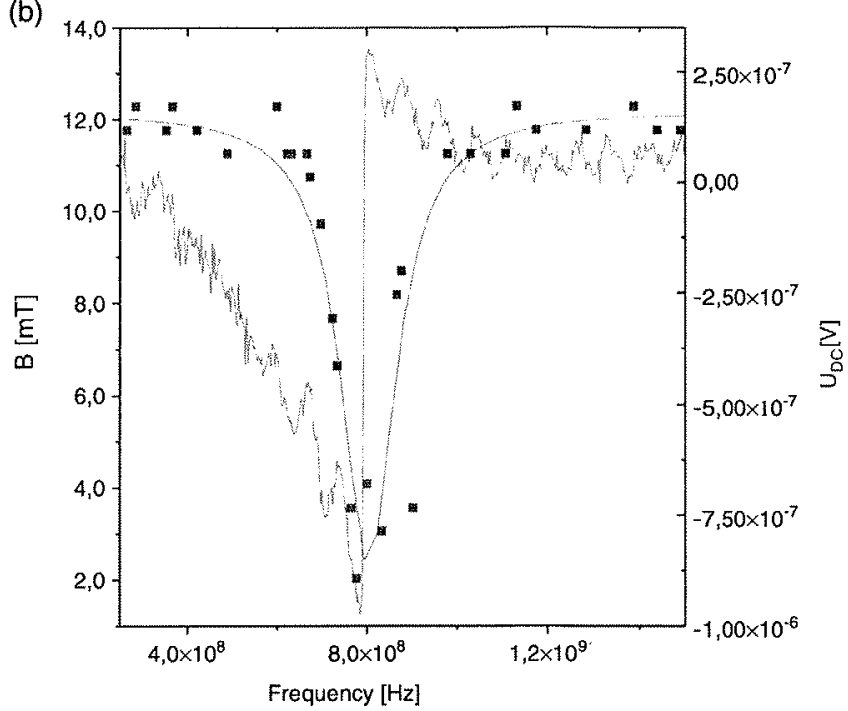

Figure 18. (a) Depinning fie $d$ (blue squares, blue line) of the transverse wall located inside the constriction as a function of frequency. A dip at $1.3 \mathrm{GHz}$ is observed. The inset shows the full frequency spectrum scanned. The red line is the DC response, with a feature at the same frequency. (b) Depinning fie d (blue squares) for a vortex wall pinned adjacent to the constriction with a dip at around $800 \mathrm{MHz}$. The blue line is a Lorentzian $\mathrm{ft}$ to the depinning data. For the vortex wall the DC response (red line) exhibits, in addition to the dip, a dispersion-like signal due to the change in phase at the resonance frequency. Reproduced with permission from [94]. Copyright 2007 by the American Physical Society.

In $f$ gures $17($ b)-(d) results of micromagnetic simulations of the wall types that we investigated are shown: (b) spin structures for a transverse wall inside the constriction; (c) a vortex wall pinned adjacent to the constriction; (d) a free vortex wall. To measure the resonance, the walls are excited with microwaves of varying frequency and then the depinning feld is measured. On resonance we expect a dip in the depinning feld, as the feld necessary to lift the oscillating quasi-particle domain wall out of the potential well is reduced.

We frst measure the depinning of a transverse wall (f gure 17(b)) by positioning the wall with a feld along the notch direction $\left(35^{\circ}\right.$, between contacts 3 and 4$)$ as detailed in [21] and then applying a perpendicular f eld along $305^{\circ}$. In fgure 18(a) (blue squares) we see that the depinning feld off-resonance is $53.7 \mathrm{mT}$ and exhibits a dip to $51.5 \mathrm{mT}$ at a resonance frequency of about $1.3 \mathrm{GHz}$. To determine the nature of this eigenmode, micromagnetic simulations were carried out and a resonant eigenmode at a frequency of $1.2 \mathrm{GH}$ was obtained. The eigenmode consists of the whole wall moving in the constriction, so that here the analogy with a quasi-particle holds very well.

Next we consider a pinned vortex wall (f gure 17(c)). From topological considerations the simplest soft eigenmode of the vortex wall is the motion of the vortex core along the diagonal connecting the two topological edge defects (indicated in fgure $17(\mathrm{c})$ ). Qualitatively this eigenmode was predicted to occur for vortex walls by Tchernyshyov et al [90. 91]. This corresponds to a frst approximation of the vortex core motion in a disc, which has been widely studied. Guslienko et al determined the frequency of the vortex core oscillatory eigenmode analytically for a disc [92], and taking the wire width as the disc diameter a frequency of $900 \mathrm{MHz}$ is predicted. Experimentally a dip in the depinning feld is observed at around $800 \mathrm{MHz}$ (f gure 18 (b), blue squares), so that the simple theoretical model yields a surprisingly good agreement, even though it is calculated for zero f eld. Micromagnetic simulations corroborate this picture, and they reveal a combination of the linear motion predicted by Tchernyshyov and the circular motion expected in a disc, resulting in an ellipsoidal motion with the long axis along the diagonal connecting the two edge defects, from which it is clear why no perfect agreement to the disc model can be expected. It is important to note that the eigenmode that leads to a reduction of the depinning $f$ eld is not a translational mode of the entire domain wall (as claimed for an unpinned wall in [93]) but the oscillation of the vortex core described above.

As the depinning feld under microwave excitation is not zero for the microwave power level chosen, the resonance frequency obtained by this method corresponds to the domain wall resonance frequency at the depinning feld. In order to determine the resonance frequencies independently and at variable felds, a homodyne detection scheme based on a DC signal was developed as described in [94] and similar schemes were presented by Moriya et al [95] and Yamaguchi et al [96]. We plot the DC response for the vortex wall pinned at the constriction in f gure $18(\mathrm{~b})$ (red line). For the vortex wall (f gure 18(b)) we see a dispersion-like signal with a change in the sign exactly at the resonance frequency, which allows us to determine the resonance from the DC signal. For the transverse wall the expected signal is much weaker since the oscillation has a smaller amplitude, as visible from the smaller change in the depinning feld compared to the vortex wall, and this is also ref ected in the smaller DC signal (f gure 18(a) (red line)). 

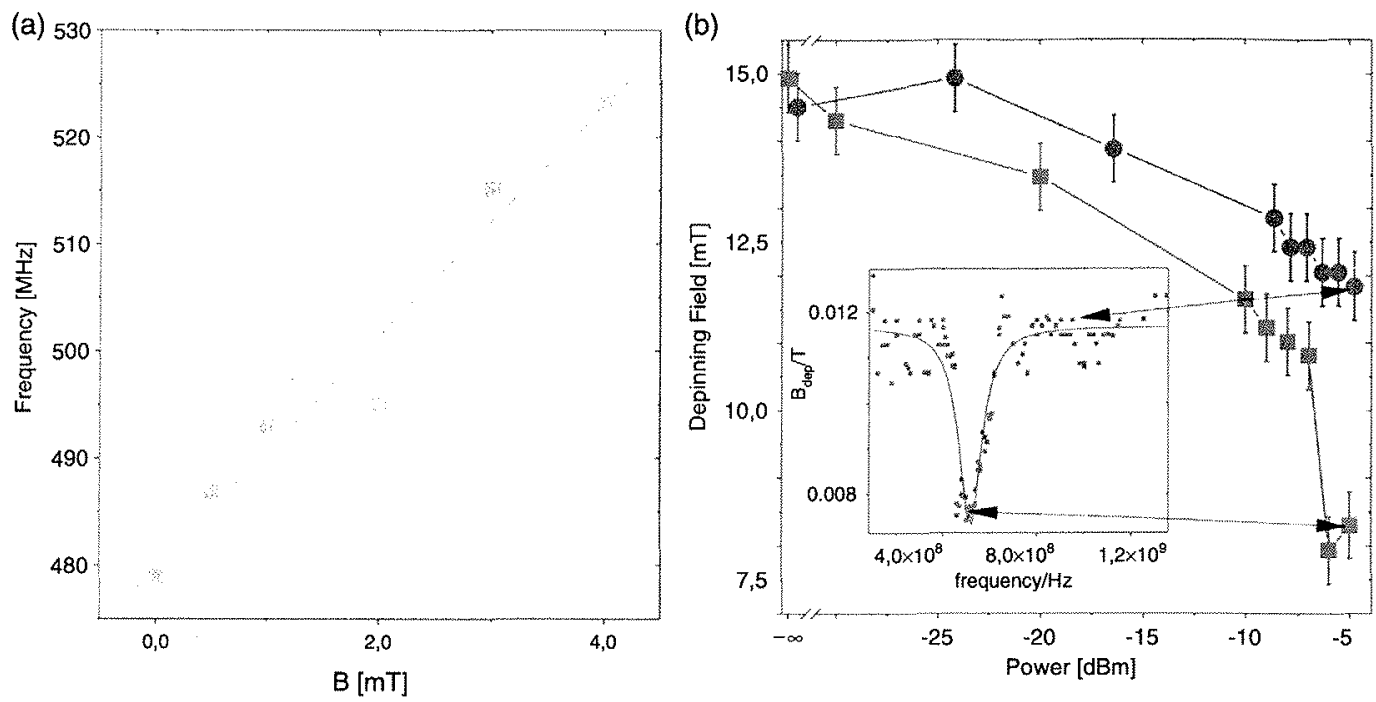

Figure 19. (a) The frequency dependence of the resonance for the free vortex wall on the strength of an applied feld exhibits a linear increase as theoretically predicted. The green line results from a linear fi. (b) Dependence of the depinning fie $\mathrm{d}$ of the free vortex wall on the injected current density (power) for current injection on-resonance ( $480 \mathrm{MHz}$, red squares) and off-resonance ( $850 \mathrm{MHz}$, blue discs). The reduction for the off-resonant case can be atributed to heating. A threshold power of $-7 \mathrm{dBm}$ is visible, which points to a strongly non-linear oseillator The inset shows the depinning spectrum corresponding to a power level of $-5 \mathrm{dBm}$. Reproduced with permission from [94]. Copyright 2007 by the American Physical Society.

The DC detection method now allows us to also study the feld dependence of the vortex core oscillation frequency. For this measurement we use a free vortex wall since the vortex core motion eigenmode is expected to be present also in a free vortex wall, where the infuence of the notch is eliminated (f gure $17(\mathrm{~d})$ ). Here the fnite wall propagation feld is due to pinning at defects such as the edge roughness. The wall resonance occurs at a lower frequency of $480 \mathrm{MHz}$. This lower frequency compared to the pinned vortex core can be understood in terms of the larger effective width of the wire, which is $200 \mathrm{~nm}$ wide, whereas in the pinned case the average width is reduced due to the constriction on one side of the wall.

In $f$ gure $19(a)$ the frequency of the DC signal (free vortex) is plotted for different external felds. A weak increase of the frequency with increasing feld is visible, which is in line with theoretical predictions [98]. For applied f elds above $7 \mathrm{mT}$ the domain wall is drawn out of the area between the contacts and no DC signal is measured. Whether the resonance frequency increases or decreases with increasing feld depends sensitively on the details of the potential well and the feld direction and we have also observed that after an initial increase of the frequency at small felds, the frequency then decreases for larger felds.

To determine the power necessary to excite the wall, we plot in fgure $19(b)$ the dependence of the depinning felds as a function of the injected power for on and off-resonance frequencies. For low injected currents there is virtually no change in the depinning $f$ eld between the different frequencies. Above a threshold power of $-7 \mathrm{dBm}$, the depinning feld starts to be strongly reduced for the resonant case, whereas the nonresonant current only weakly inf uences the depinning $f$ eld due to heating. This pronounced non-linear behaviour points to a strongly non-harmonic potential.
Now that we have established the non-linear behaviour of the domain wall oscillation potential, we would like to determine directly the deviation of the potential $U(x)$ from the harmonic oscillator potential. To achieve this, we can deduce directly the shape of the potential well from the power dependence of the resonance frequency. For a symmetric potential well, the period of the oscillation as a function of the energy $E$ stored in the system with an effective mass $m$ is given by [99]:

$$
T(E)=2 \sqrt{ } 2 m \int_{0}^{E} \mathrm{~d} x_{2} \quad \begin{gathered}
\mathrm{d} U \\
\mathrm{~d} U
\end{gathered}
$$

Integrating and rearranging the terms yields the integral equation (5). We can now directly calculate the shape of the potential by numerically integrating the elliptic integral equation (5):

$$
x(U)=\underbrace{1}_{2 \pi \sqrt{ } 2 m} \int_{0}^{U} \mathrm{~d} E \begin{gathered}
T(E) \\
\sqrt{ } U-E
\end{gathered} .
$$

From the resonance frequency as a function of the power, we obtain the oscillation period and, since the energy of an oscillator is proportional to the square of the driving force, also as a function of the energy in the system. So the energy is proportional to the power applied and the energy dependence of the oscillation period is presented in $f$ gure 20 (large blue disks). To determine $x(U)$ through equation (5), a third-order polynomial interpolation has been $f$ tted to the data (small blue dots).

Integrating equation (5) over the interpolated data shown in $f$ gure 20 we obtain the shape of the potential of the domain wall oscillator for the free vortex wall and this is shown in fgure 21. The absolute width is determined as described in [21]. The blue dots are calculated from the measured 


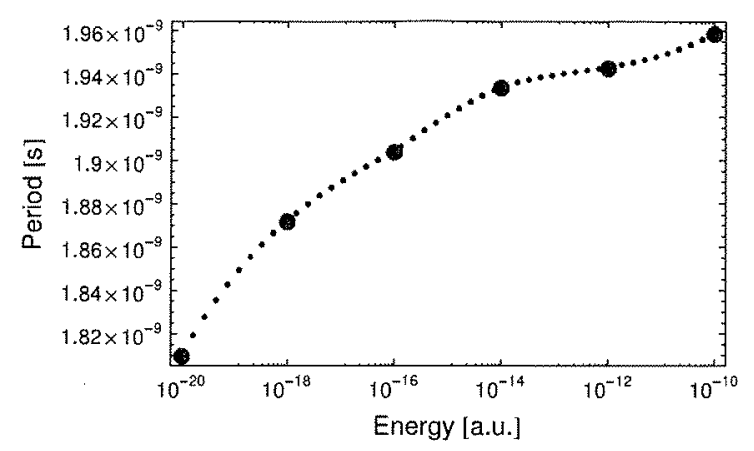

Figure 20. Period of the domain wall oscillator for different energies. The large blue dots are the measured values and the small blue dots the third-order interpolation used for the numerical integration. Reproduced partly with permission from [124]

oseillator periods and the red line indicates the parabolic part of the potential well. As expected, the domain wall potential $f$ attens far away from the origin, indicating the non-harmonic (non-linear) contribution.

\subsection{Further details of pinning behaviour}

While the instructive cases discussed above serve to convey a general idea of the domain wall pinning mechanisms, further combinations of wall types and pinning sites have been studied. The depinning felds presented so far were applied perpendicular to the wall position, which yields a minimum in the depinning f eld. If the feld is applied at an angle, the component of the feld acting to depin the wall is reduced, which yields an increase of the total feld necessary to depin the wall. Such an angular dependence has been measured in detail and good agreement with micromagnetic simulations is found [100]. In particular the minimum in the depinning f eld allows one to deduce the exact wall position [100].

So far we have looked only at the depinning, i.e. moving a wall away from the notch. By measuring the felds necessary to move a transverse wall into a notch, Petit et al found [101] that there is a small energy barrier that separates the fat potential away from the notch and the potential well (as seen in the schematic of the potential landscape in f gure 14(a)). Furthermore, we have limited ourselves to studying constrictions due to a single notch pointing upwards with a transverse wall that is also pointing upwards. For a single notch with a given direction, the transverse wall can be pointing up or down and the circulation direction of the vortex wall can be clockwise or counter-clockwise; the potential landscapes for all these cases were ascertained in [102]. In addition to the different wall types, one can also vary the notches by introducing a notch from either side of the structure or even use multiple notches. The inf uence of protrusions rather than notches has also been investigated [101] and sharp bends have been used to pin walls $[103-105]$. When walls are injected from an injection pad into a wire, pinning can occur at the position where the pad meets the wire due to the abrupt change in the geometry $[106,107]$ and by choosing the injection feld direction carefully, the wall spin structure can be set [108].

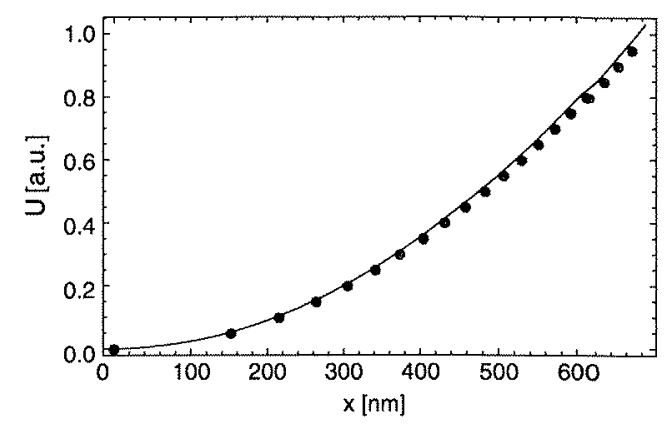

Figure 21. Domain wall potential $U(x)$. The blue dots are calculated from the measured oscillator periods and the red line indicates the parabolic part of the potential well. As expected the domain wall potential f attens far away from the origin. Reproduced partly with permission from [124].

Finally the resonant behaviour of a domain wall in a potential well can be probed not only by injecting an $\mathrm{AC}$ current but also by using short well-timed pulses, with a distance between pulses that corresponds to the resonance as shown by Thomas et al [109]. This also allows one to describe the domain wall by a phase in addition to a position [110].

In summary, geometrical variations generate a potential landscape for a domain wall that depends on the details of the geometrical variation (notch, protrusion, etc) and the wall type. Using different types of domain walls and constrictions with different geometries, we can engineer well-defi ed pinning potentials, which can then act as stable positions for domain walls in applications.

\section{Domain wall propagation}

So far we have looked at static domain walls or domain walls carrying out small oscillations. The next step is to look at the dynamics of the wall propagation. Since this is a very active feld of research with many open questions, we will only give a brief overview of the feld and discuss rather qualitatively the behaviour of the wall velocity for a ID model system. For further information and a more quantitative and detailed discussion for more realistic wall spin structures, the reader is advised to consult the specialized literature that we refer to.

In general, the magnetization dynamics is governed by the Landau-Lifshitz Gilbert equation augmented by spin torque terms with the f rst term accounting for $f$ eld effects, the second for damping and the last two terms accounting for effects of an injected current $[3,111]$ :

$$
\begin{gathered}
\partial \mathbf{m}(t)=-\gamma_{0} \mathbf{m} \times \mathbf{H}_{\mathrm{eff}}+\alpha \mathbf{m} \times \underbrace{\partial \mathbf{m}(t)}_{\partial t} \\
\partial t \\
-\underbrace{(\mathbf{u} \cdot \nabla) \mathbf{m}}_{\text {adiabatic }}+\underbrace{\beta \mathbf{m} \times[(\mathbf{u} \cdot \nabla) \mathbf{m}]}_{\text {non-adiabatic }}
\end{gathered}
$$

with $\mathbf{H}_{e f f}$ the effective feld, $\gamma_{0}$ the gyromagnetic ratio, $\alpha$ the damping constant and the effective velocity $\mathbf{u}$ given by $\mathbf{u}={ }_{2 e M}^{g P \mu_{B}} \mathbf{j}$ ( $P$ is the spin polarization, the electron charge $e$, the saturation magnetization $M_{s}$, the current density $j$ and the $g$-factor) [26]. The non-adiabaticity parameter is in one theory [27] given by $\beta=\left(\lambda_{\mathrm{ex}} / \lambda_{\mathrm{sf}}\right)^{2}$ (ratio of the exchange 


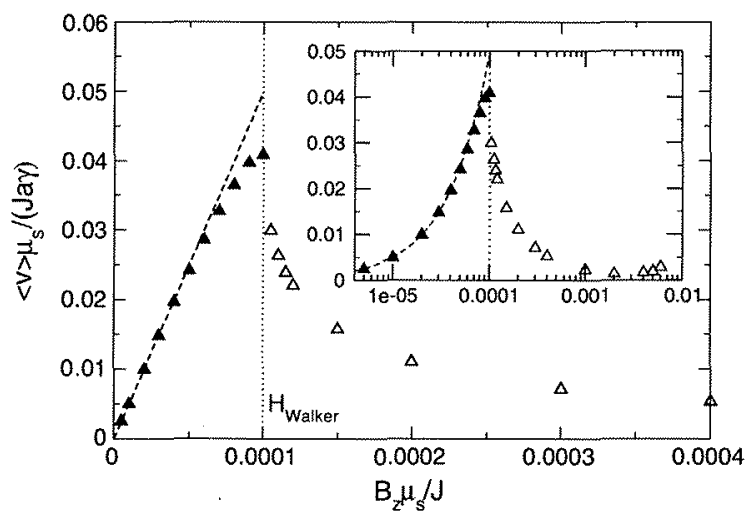

Figure 22. Simulation of the wall velocity as a function of the applied feld for a one-dimensional spin chain (for details of the simulation and the units used, see [1 17]). The velocity increases linearly up to a critical feld (Walker feld). Above this feld. transtormations set in, which reduce the velocity. For even higher fie ds, the velocity increases again as seen in the inset, which is plotted on a logarithmic feld scale.

length and the spin-fip length) but the theory of the nonadiabaticity is still hotly debated.

The frst two terms account for precession and damping, whereas the third term is the adiabatic spin torque term due to the injected current. The fourth term is often called non-adiabatic spin torque term but it contains not only contributions due to non-adiabatic transport but also due to spin relaxation [112]. Since we limit ourselves here to a brief overview, the reader is referred to reviews and more specialized literature $[26,27,54,55,112-114]$ for an in-depth discussion of the effects of these terms.

\subsection{Field-induced wall motion}

When a feld is applied to a system, a domain wall will feel a change in the potential, and this can for instance change the energetically favourable wall position. If the feld is suff ciently high to overcome the local pinning, the wall will move to a new equilibrium position. In a smooth wire with no pinning sites this means that even very small felds move the domain walls. The actual feld-induced propagation process was studied in an analytical model by Walker and Schryer [115] for $180^{\circ}$ walls with an easy axis. They found that walls should move with a velocity $\mathbf{v}=(\gamma \Delta / \alpha) \cdot \mathbf{H}$ with $\gamma$ the gyromagnetic ratio, $\Delta$ the wall width and $\alpha$ the damping constant. As the feld is increased the wall deforms (the details depending on the wall type) and at a critical feld $H_{\text {Walker, }}$ the so called Walker breakdown occurs, where the wall spin structure transforms [115]. As an example the velocity of a head-tohead transverse wall in a one-dimensional wire as a function of the applied feld is shown in fgure 22. As theoretically expected, the wall velocity scales linearly with the feld up to the Walker breakdown feld, where wall transformations set in. These transformations then reduce the wall velocity and only at much higher felds do the wall velocities start to increase again. A very thorough theoretical investigation

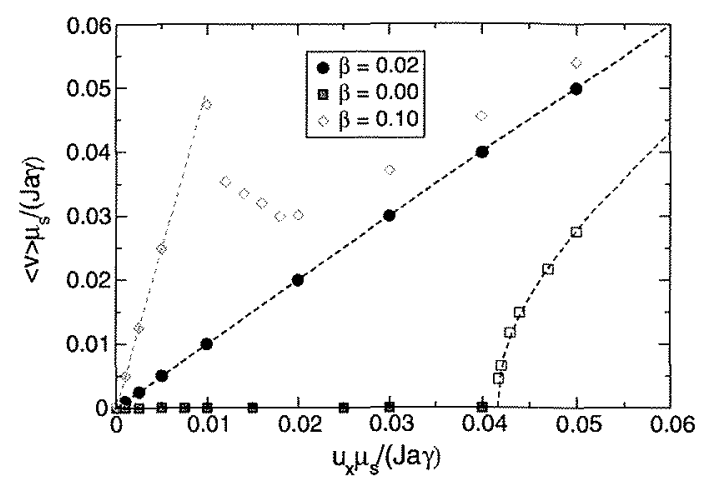

Figure 23. Simulation of the wall velocity as a function of the injected current density $j$ (for details of the simulation and the units used, see [117]). The damping constant used is $\alpha=0.02$ and three different values of the non-adiabaticity parameter $\beta$ are used: $\beta=0$ (red empty squares), $\beta=0.02=\alpha$ (black discs) and $\beta=0.1$ (green diamonds).

of the behaviour of realistic transverse and vortex head-tohead domain wall spin structures under applied felds was carried out recently [116]. In general a similar behaviour was found with an increase in the velocity up to a Walker $f$ eld. For higher felds, depending on the geometry and f eld, different transformations including vortices and antivortices were found to occur, which are described in detail in [116]. Theoretically the inf uence of roughness on wall propagation was also investigated and rough wires were found to suppress the vortex nucleation [15].

Experimentally, the feld-induced wall motion of head-tohead domain walls was studied by a number of groups. In GMR structures, wall velocities were determined in [13] and indirectly by varying the pulse length in [14]. More recently direct dynamic measurements using optical Kerr effect were carried out by Beach et al [11] and MR measurements were carried out by Hayashi et al [12]. Their experimental results agree well with the theoretical predictions and they can even detect the periodic transformations that set in above the Walker breakdown [12].

\subsection{Current-induced wall motion}

For the current-induced wall propagation, the situation is depicted in foure 23 [117]. Here, the behaviour depends on the non-adiabaticity parameter $\beta$. For the purely adiabatic case ( $\beta=0$, red squares) a high critical current density $j_{\mathrm{c}}$ is observed as predicted by Thiaville [26] and Zhang [27] and the velocity follows a $\sqrt{ }\left(j^{2}-j_{c}^{2}\right)$ behaviour [28] and approaches the effective velocity $u$ (see equation $(6)$ ) for large current densities. If non-adiabatic transport or spin relaxation exist $(\beta \neq 0)$, the critical current density is reduced to zero for an ideal wire and the velocity increases, at f rst linearly with current density. For the case that $\beta=\alpha$ (black discs) the velocity increases, always linearly (velocity equals $u$ ), and the wall is not deformed. For $\beta \neq 0$ and $\beta \neq \alpha$ (green diamonds) the velocity scales as $\beta \cdot u$ up to a peak at the Walker current density. Above this, wall transformations set 
in analogous to the feld-induced wall motion case and for high current densities the velocity approaches $u$.

The types of wall transformations occurring for headto-head transverse and vortex walls have been studied theoretically in detai] [118] and include vortices and antivortices depending on the geometry and current densities used.

Experimentally current-induced wall motion has been studied by a large number of groups (for an overview see references in $[10,12,16-18,79,119-121])$. Dynamic measurements of the velocity have been carried out by Hayashi et al for the case of a domain wall, which is dynamically generated. Here, high velocities exceeding $100 \mathrm{~m} \mathrm{~s}^{-1}$ have been reported [121] and combining the currents with fie ds, the velocity enhancement due to currents has been measured [120]. Starting with a domain wall at rest, slower velocities have been observed for pure current-induced wall motion $[16,17,122]$ and for suff ciently high current densities periodic wall transformations have been imaged, which points to the fact that $\beta \neq \alpha$ for the Permalloy samples used in these studies $[18,123]$.

\section{Conclusions}

In conclusion. we have presented an overview of the spin structure of head-to-head domain walls in magnetically soft $f$ at elongated nanostructures such as wires. We have extracted the corresponding room temperature phase diagrams with two phase boundaries between vortex walls for thick and wide as well as ultrathin rings and transverse walls for thin and narrow rings. For very wide structures, additional more complicated wall types are found and discussed.

Using temperature-dependent measurements, we have observed a thermally activated switching from transverse walls established at room temperature to vortex walls. This gives direct experimental evidence that transverse and vortex walls can both constitute local energy minima and are separated by an energy barrier, which can be overcome thermally.

We have mapped the stray feld of a domain wall directly using off-axis electron holography, and we fnd that the feld strength falls off with a $1 / r$-dependence. For interacting domain walls we observe a transition from a transverse to a vortex spin structure with increasing edge-to-edge spacing. By correlating this transition with the measured stray f eld, we are able to obtain the energy barrier height distribution for vortex core nucleation in Co samples.

The quasi-particle behaviour of domain walls at geometrical variations, such as constrictions, is discussed in detail. In particular the potential landscape that constrictions generate for transverse and vortex walls is determined including the quantitative width and depth of the attractive potential wells. To determine the well curvature, dynamic resonant wall oscillations are studied and from the power dependence of the resonance frequency the exact shape of the potential well is extracted.

Large scale domain wall motion due to felds and currents is briefy reviewed and the velocity versus feld (respectively current) diagrams are discussed including the
Walker breakdown. For the current-induced case, the different behaviour depending on the non-adiabaticity is presented.

While this overview is envisaged to give the reader an introduction to the exciting properties of head-to-head domain walls it is far from exhaustive and some exciting recent $f$ ndings such as the inf uence of walls on spin wave phases [125] are beyond the scope of the review. In particular the areas of $f$ eldand current-induced wall dynamics are still developing at a fast pace so that this review can only provide a rough overview of the state of the research at this moment.

Nonetheless for the main properties of head-to-head domain walls a reasonably comprehensive review is attempted and it is hoped that this review will be found useful as a frst introduction to this exciting topic and stimulate further research.

\section{Acknowledgments}

Support by the Deutsche Forschungsgemeinschaft through SFB 513, SFB 767, project KL1811, the Landesstiftung Baden-Württemberg, the EPSRC (UK), the EC through the 6th Framework Programme, and by the EU througl the European Regional Development Fund (Interreg III A Programme) as well as the European Research Council (Starting Independent Researcher Grant) is greatly acknowledged. A large number of PhD students, postdocs and colleagues have contributed to the various aspects of this work and all their support. help and encouragement is gratefully acknowledged. Helpful comments and proof reading of various parts of the manuscript were provided by $T$ Moore, U Nowak, C A F Vaz and L J Heyderman. Continuous support by J A C Bland' and $U$ Rüdiger was crucial to the success of the work at many stages. The unpublished f gure 22 was kindly provided by C Schieback.

\section{References}

[1] Kläui M and Vaz C A F 2007 Handbook of Magnetism and Advanced Magnetic Materials vol 2, ed H Kronmüller and S S P Parkin (Chichester: Wiley)

[2] Hubert A and Schäfer R 1998 Magnetic Domains-The Analysis of Magnetic Microstructures (Berlin: Springer)

[3] Landau L D and Lifshitz E 1935 Phys. Z. Sowjetunion 8153

[4] Néel L 1955 C. R. Hebd. Séances Acad. Sci. 249533

[5] Bloch F 1932 Z. Phys. A 74295

[6] Thiaville A and Nakatani Y 2006 Spin Dynamics in Confined Magnetic Structures vol III, ed B Hillebrands and K Ounadjela (Berlin: Springer)

[7] Fert A and Piraux L 1999 J. Magn. Magn. Mater. 200338

[8] Kim G-H et al 1998 Phys. Rev. B 5812138

[9] Brooke J et al 2001 Nature 413610

[10] Kläui M et al 2005 Appl. Phys. Lett. 87102509

111] Beach G S D, Nistor C, Knutson C, Tsoi M and Erskine J L 2005 Nat. Mater. 4741

[12] Hayashi M et al 2007 Nat. Phys. 321

[13] Ono T et al 1999 Science 284468

[14] Atkinson D, Allwood D A, Xiong G, Cooke M D, Faulkner C C and Cowburn R P 2003 Nat. Mater. 285

[15] Nakatani Y, Thiaville A and Miltat J 2003 Nat. Mater. 2521

[16] Yamaguchi A, Ono T, Nasu S, Miyake K, Mibu K and Shinjo T 2004 Phys. Rev. Lett. 92077205

1 Deceased 
[17] Kläui M, Vaz C A F, Bland I A C, Wernsdorfer W, Faini G, Cambril E, Heyderinan L J, Nolting F and Rüdiger U 2005 Phys. Rev. Lett. 94106601

[18] Kläui M, Jubert P-O, Allenspach R, Bischof A, Bland J A C, Faini $G$, Rüdiger U, Vaz C A F, Vila L and Vouille C 2005 Phys. Rev. Lett. 95026601

[19] Levy P M and Zhang S 1997 Phys. Rev. Lett. 79 5110

[20] Ebels U, Radulescu A, Henry Y, Piraux L and Ounadjela K 2000 Phys. Rev. Lett. 84983

[21] Kläui M, Vaz C A F, Rothman J, Bland J A C, Wernsdorfer W, Faini $G$ and Cambril E 2003 Phys. Rev. Lett. 90097202

[22] Rüdiger U, Yu J, Zhang S, Kent A D and Parkin S S P 1998 Phys. Rev. Lett. 805639

[23] Kent A D, Yu J, Rüdiger U and Parkin S S P 2001 J. Phys.: Condens. Matter 13 R 46 I

[24] Viret M et al 1996 Phys. Rev. B 538464

[25] Marrows C et al 2005 Adv. Phys. 54585

[26] Thiaville A, Nakatani Y, Miltat J and Suzuki Y 2005 Europhys. Lett. 69990

[27] Zhang S and Li Z 2004 Phys. Rev. Lett. 93127204

[28] Tatara $G$ and Kohno H 2004 Phys. Rev. Lett. 92086601

[29] Yamaguchi A, Nasu S, Tanigawa H, Ono T, Miyake K, Mibu K and Shinjo T 2005 Appl. Phys. Lett. 86012511

[30] Laufenberg M, Bührer W, Bedau D, Melchy P-E, Kläui M, Rüdiger U, Vila L, Faini G, Vaz C A F and Bland I A C 2006 Phys. Rev. Lett. 97046002

[31] Kazantseva N, Wieser R and Nowak U 2005 Phys. Rev. Lett. 94037206

[32] Junginger F et al 2007 Appl. Phys. Lett. 90132506

[33] Bulacvskii L N and Ginzburg V L 1964 Sov. Phys,_JETP 18530

[34] Garanin D A 1991 Physica A 172470

[35] Bland J A C, Lopez-Diaz L, Kläui M and Rothman J 2002 Patent Specification ep 1307891

[36] Allwood D A, Xiong G, Faulkner C C, Atkinson D, Petit D and Cowburn R P 2005 Science 309 1688

[37] Allwood D A, Xiong G, Cooke M D, Faulkner C C, Atkinson D, Vernier N and Cowburn R P 2002 Science 2962003

[38] Parkin S S P 2004 US Patent Specification 6,834,005 Parkin S S P 2004 US Patent Application 10/984,055

[39] Parkin S S P et al 2008 Science 320190

[40] Cowburn R 2004 Patent Application pct/gb2003/001266 Cowburn R 2004 Patent Application pct/gb2004/000840

[41] Rothman I, Kläui M, Lopez-Diaz L, Vaz C A F, Bleloch A, Bland J A C, Cui Z and Speaks R 2001 Phys. Rev. Lett. 861098

[42] Li S P, Peyrade D, Natali M, Lebib A, Chen Y, Ebels U, Buda L D and Ounadjela K 2001 Phys. Rev. Lett. 861102

[43] Kläui M, Vaz C A F, Lopez-Diaz L and Bland I A C 2003 J. Phys.: Condens. Matter $15 \mathrm{R} 985$

[44] Martin J I et al 2002 J. Magn. Magn. Mater. 256419

[45] Backes D et al 2006 Microelectron. Eng. 831726

[46] Heyderman L J, David C, Kläui M, Vaz C A F and Bland J A C 2003 J. Appl. Phys. 9310011

[47] Kronmüller $\mathrm{H}$ and Parkin S S P (ed) 2007 Handbook of Magnetism and Advanced Magnetic Materials vol 3 (Chichester: Wiley)

[48] Stöhr J, Wu Y, Hermsmeier B D, Samant M G, Harp G R, Koranda S, Dunham D and Tomner B P 1993 Science 259658

[49] Stöhr J and Anders S 2000 IBM J. Res. Dev. 44535

[50] Tonomura A 1992 Adv. Phys. 1159

[51] Dunin-Borkowski R E, McCartney M R, Kardynal B Parkin S S P, Scheinfein M R and Smith D J 2000 J. Microsc. 200187

[52] Chapman J et al 1989 Mater. Sci. Eng. B 3355

[53] Kronmüller H and Fähnle M 2003 Micromagnetism and the Microstructure of Ferromagnetic Solids (Cambridge: Cambridge University Press)
[54] Miltat J and Donahue M J 2007 Handbook of Magnetism and Advanced Magnetic Materials vol 2, ed H Kronmüller and S S P Parkin (Chichester: Wiley)

[55] Schref T et al 2007 Handbook of Magnetism and Advanced Magnetic Materials vol 2, ed H Kronmüller and S S P Parkin (Chichester: Wiley)

[56] McMichael R D and Donahue M J 1997 IEEE Trans. Magn. 334167

[57] Wachowiak A, Wiebe J, Bode M, Pietzsch $\mathrm{O}$, Morgenstern $M$ and Wiesendanger R 2002 Science 298577

[58] Junginger F et al 2008 Appl. Phys. Lett. 92112502

[59] Feldtkeller E et al 1965 Phys. Kondens. Mater 48

[60] Nakatani Y, Thiaville A and Miltat J 2005 J. Magn. Magn. Mater: $290 / 291750$

[61] Backes Det al 2007 Appl. Phys. Lett. 91112502

[62] Kläui M, Vaz C A F, Bland I A C, Heyderman L J, Nolting F, Pavlovska $A$, Bauer E, Cherif $S$, Heun $S$ and Locatelli A 2004 Appl. Phys. Lett. 855637

[63] Laufenberg M et al 2006 Appl. Phys. Lett. 88052507

[64] Yoo Y G, Kläui M, Vaz C A F, Heyderman L J and Bland J A C 2003 Appl. Phys, Lett. 822470

[65] Bromwich T J et al 2006 J. Appl. Phys. $9908 \mathrm{H} 304$

[66] Kläui Met al 2004 Physica B 343343

[67] The oommf package is available at http//math nist gov/ oommt

[68] Heyne L et al 2008 J. Appl. Phys. 103070928

[69] Castano F et al 2003 Phys. Rev. B 67184425

[70] Uhlig T and Zweck J 2004 Phys. Rev. Lett. 93047203

[71] Zhu X, Grütter P, Metlushko V, Hao Y, Castano F J, Ross C A, Ilic B and Smith HI 2003 J. Appl. Phys. 938540

[72] Hashim I, Joo H S and Atwater H. A 1994 Surf. Rev. Lett. 2427

[73] Vaz C A F, Kläui M, Heyderman L J, David C, Nolting F and Bland J A C 2005 Phys. Rev. B 72224426

[74] Kläui M, Rüdiger U, Vaz C A F, Bland J A C, Cherif $S$, Locatelli A, Heun S, Pavlovska A, Baner E and Heyderman L J 2006 J. Appl. Phys. 99086308

[75] Hempe E-M et al 2007 Phys. Status Solidi a 2043922

[76] Kläui M et al 2006 Appl. Phys. Lett. 88232507

[77] Park M H, Hong Y K, Choi B C, Donahue M J, Han H and Gee S H 2006 Phys. Rev. B 73094424

[78] McGrouther D et al. 2007 Appl. Phys. Lett. 9122506

[79] Meier G et al 2007 Phys. Rev. Lett. 98187202

[80] Nozaki Y et al 1999 Japan. J. Appl. Phys. 386282

[81] Bruno P 1999 Phys. Rev. Lett. 832425

[82] Jubert P-O, Allenspach R and Bischof A 2004 Phys. Rev. B 69220410

[83] Heyderman L J, Kläui M, Schäublin R, Rüdiger U, Vaz C A F, Bland $J A C$ and David C 2005 J. Magn. Magn. Mater. $290 / 29186$

[84] Laufenberg M et al 2006 Appl. Phys. Lett. 88212510

[85] Cowburn R P, Koltsov D K, Adeyeye A O, Welland ME and Tricker D M 1999 Phys. Rev. Lett. 831042

[86] Kläui M, Vaz C A F, Bland J A C and Heyderman L. J 2005 Appl. Phys. Lett. 86032504

[87] McCaig M 1977 Permanent Magnets in Theory and Practice Ist edn (London: Pentech)

[88] Faulkner C et al 2004 J. Appl. Phys. 956717

[89] Kläui M, Vaz C A F, Bland J A C, Wernsdorfer W, Faini G and Cambril E 2002 Appl. Phys. Lett. 81108

[90] Tchernyshyov O et al 2005 Phys. Rev. Lett. 95197204

[91] Youk Het al 2006 J. Appl. Phys. 9908B 10।

[92] Guslienko K et al 2002 J. Appl. Phys. 918037

[93] Saitoh E, Miyajima H, Yamaoka T and Tatara G 2004 Nature 432203

[94] Bedau D et al 2007 Phys. Rev. Lett. 99146601

[95] Moriya R 2007 Presentation J14 2 at the APS March Mtg; Nature Phys. doi:10.1038/nphys 936 
[96] Yamaguchi A et al 2007 Appl. Phys. Lett. 91132500

[97] Laufenberg M 2006 PhD Thesis Universităt Konstanz

[98] Novosad V et al 2005 Phys. Rev. B 722445

[99] Landau L D and Lifshitz E M 1982 Theoretical Physics-Mechanics (New York:

Butterworth-Heinemann)

[100] Bedau D et al 2007 J. Appl. Phys. 101091509

[101] Petit D et al 2007 Preprint $0711.5026 \mathrm{~V}$

[102] Hayashi M et al 2006 Phys. Rev. Lett. 97207205

[103] Zhu X et al. 2003 J. Appl. Phys. 937059

[104] Libal A et al 2005 J. Appl. Phys. 9883904

[105] Brownlie C et al 2006 J. Appl. Phys. 10033902

[106] Kläui M, Vaz C A F, Lapicki A, Suzuki T, Cui Z and Bland I A C 2004 Microelectron. Eng. 73/74785

[107] Thomas L, Retner C, Hayashi M, Samant M G, Parkin S S P Doran A and Scholl A 2005 Appl. Phys. Lett. 87262501

[108] Atkinson D et al 2008 Appl. Phys. Lett. 9222510

[109] Thomas L et al 2007 Science 3151553
[110] Thomas L et al 2006 Nature 443197

[111] Brown W F Jr 1963 Micromagnetics (New York Interscience)

[112] Tatara G et al 2007 J. Phys. Soc, Japan 7654707

[113] Barnes S E and Mackawa S 2006 Concepts in Spin Electronics ed S Maekawa (Oxford: Oxford University Press)

[114] Tserkovnyak Y et al 2008 J. Magn. Magn. Mater. 3201282

[115] Schryer N L and Walker L R 1974 J. Appl. Phys. 455406

[116] Lee J-Y et al 2007 Phys. Rev. B 76184408

[117] Schicback C et al 2007 Eur. Phys. J. B 59 429

[18] Seo S-M et al 2007 Appl. Phys. Lett. 90252507

[119] Marrows C H 2005 Adv. Phys. 54585

[120] Beach G S D et al 2007 Phys. Rev. Lett. 9757203

[121] Hayashi $M$ et al 2007 Phys. Rev. Lett. 9837204

[122] Jubett P.O et al 2006 J. Appl. Phys. 99089523

[123] Heyne L et al 2008 Phys. Rev. Lett. 10066603

[124] Bedau D 2007 PhD Thesis Universität Konstanz (Bedau D et al, unpublished)

[125] Hertel R et al 2004 Phys. Rev. Lett. 93257202 